\title{
Boundedness and applications of singular integrals and square functions: a survey
}

\author{
Steve Hofmann • Alan McIntosh
}

Received: 15 September 2011 / Accepted: 23 September 2011 / Published online: 11 October 2011 (C) The Author(s) 2011. This article is published with open access at SpringerLink.com

\begin{abstract}
We present a survey of certain aspects of the theory of singular integrals and square functions, with emphasis on $L^{2}$ boundedness criteria and recent applications in partial differential equations.
\end{abstract}

Mathematics Subject Classification (2000) Primary 42B20 - 42B25;

Secondary $35 \mathrm{~J} 25 \cdot 35 \mathrm{~J} 55 \cdot 47 \mathrm{~F} 05 \cdot 47 \mathrm{~B} 44$

\section{Contents}

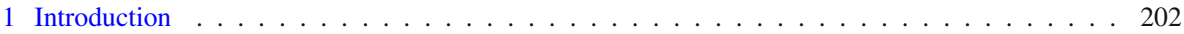

1.1 Singular integrals . . . . . . . . . . . . . . . . . . . . 202

1.2 Square functions . . . . . . . . . . . . . . . . . . . . . . 206

1.3 Notation . . . . . . . . . . . . . . . . . . . . . . . . . . . 209

$2 L^{p}$ and endpoint theory of SIOs and square functions . . . . . . . . . . . . . . 210

$3 L^{2}$ boundedness criteria . . . . . . . . . . . . . . . . . . . . . . . . . . . . . 214

3.1 The convolution case . . . . . . . . . . . . . . . . . . . . . . 214

3.2 The non-convolution case . . . . . . . . . . . . . . . . . . . . 216

Communicated by Neil Trudinger.

Steve Hofmann was supported by the National Science Foundation; Alan McIntosh was supported by the Australian Government through the Australian Research Council.

S. Hofmann

Department of Mathematics, University of Missouri, Columbia, MO 65211, USA

e-mail: hofmann@math.missouri.edu

A. McIntosh $(\varangle)$

Centre for Mathematics and its Applications, Mathematical Sciences Institute,

Australian National University, Canberra, ACT 0200, Australia

e-mail: alan.mcintosh@anu.edu.au 
4 Local $T b$ theorems and applications . . . . . . . . . . . . . . . . . . . . . . . . 225

4.1 Local $T b$ theorems for square functions . . . . . . . . . . . . . . . . . . . . . . . . . . . . . . . . . . . . . . . . . . . . . . . . . . .

4.2 Application to the Kato square root problem . . . . . . . . . . . . . . . . . . 233

5 Further results and recent progress . . . . . . . . . . . . . . . . . . . . . . . 238

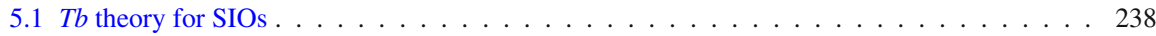

5.2 Local $T b$ theory for square functions and applications . . . . . . . . . . . . . . . . . . . 240

References . . . . . . . . . . . . . . . . . . . . . . . . . . . 240

\section{Introduction}

We survey those aspects of the theory of singular integral operators which have been obtained since the pioneering work of Zygmund, Calderón and Mikhlin, concerning the Calderón program as developed by Coifman and Meyer. Key results in this development include the Calderón commutator theorem, $L^{2}$ bounds on the higher commutators and on the Cauchy integral on Lipchitz curves, the solutions of the Painlevé problem on analytic capacity and the Kato square root problem for elliptic operators, along with further applications to analytic capacity and partial differential equations.

Our emphasis is on $L^{2}$ boundedness criteria for singular integrals, commonly known as $T 1, T b$ and local $T b$ theorems, which have arisen from and contributed to the abovementioned program. We conclude the survey with a discussion of some recent progress and applications.

For the classical theory of singular integrals and square functions, we refer the reader to the excellent monographs of Stein [86,87], and of Christ [32].

\subsection{Singular integrals}

A singular integral operator (SIO) in $\mathbb{R}^{n}$ (in the generalized sense of Coifman and Meyer) [37], is a linear mapping $T$ from test functions $\mathcal{D}\left(\mathbb{R}^{n}\right):=C_{0}^{\infty}\left(\mathbb{R}^{n}\right)$ into distributions $\mathcal{D}^{\prime}\left(\mathbb{R}^{n}\right)$, which is associated to a Calderón-Zygmund kernel $K(x, y)$, in the sense that

$$
\langle T \varphi, \psi\rangle=\iint \psi(x) K(x, y) \varphi(y) d y d x
$$

whenever $\varphi, \psi \in C_{0}^{\infty}\left(\mathbb{R}^{n}\right)$ with disjoint supports. A Calderón-Zygmund kernel is one which satisfies the standard size and Hölder bounds

$$
\begin{aligned}
& |K(x, y)| \leq C|x-y|^{-n} \quad \text { and } \\
& |K(x, y+h)-K(x, y)|+|K(x+h, y)-K(x, y)| \leq C \frac{|h|^{\alpha}}{|x-y|^{n+\alpha}}
\end{aligned}
$$

for some $\alpha \in(0,1]$, whenever $2|h| \leq|x-y|$. For now, let us take the point of view that $K: \mathbb{R}^{n} \times \mathbb{R}^{n} \backslash\{x=y\} \rightarrow \mathbb{C}$, although in the sequel we shall also mention the case that the range of $K$ is, more generally, a Hilbert space. 
We remark that, given a closed cube $Q \subset \mathbb{R}^{n}, T$ extends to a bounded linear mapping from $L^{2}(Q)$ into $L^{2}\left(\mathbb{R}^{n} \backslash Q\right)$, with the representation

$$
T f(x)=\int_{Q} K(x, y) f(y) d y
$$

for all $f \in L^{2}(Q)$ and all $x \in \mathbb{R}^{n} \backslash Q$. Indeed, this follows readily from the kernel estimate (1.2), and the Hardy inequality

$$
\int_{\mathbb{R}^{n} \backslash Q}\left|\int_{Q} \frac{1}{|x-y|^{n}}\right| f(y)|d y|^{2} d x \leq C_{n} \int_{\mathbb{R}^{n}}|f(x)|^{2} d x,
$$

along with (1.1) and a density argument.

The theory can be extended to settings other than Euclidean space, and there are worthwhile reasons for doing so, but for most of this survey we shall just consider functions defined on $\mathbb{R}^{n}$, for the sake of simplicity of exposition.

Let us now mention several examples. The Hilbert transform

$$
H f(x):=p \cdot v \cdot \frac{1}{\pi} \int_{\mathbb{R}} \frac{1}{x-y} f(y) d y:=\lim _{\varepsilon \rightarrow 0} \frac{1}{\pi} \int_{|x-y|>\varepsilon} \frac{1}{x-y} f(y) d y,
$$

relates the real and imaginary parts of a holomorphic function $F$ in the half-space $\mathbb{C}_{+}:=\mathbb{R}_{+}^{2}:=\{(x, t) \in \mathbb{R} \times(0, \infty)\}$, by the formula $H(\Re e F(\cdot, t))=-\Im m F(\cdot, t)$, assuming adequate integrability of $F$ on horizontal slices (say $F(\cdot, t)$ is uniformly in $L^{p}(\mathbb{R})$ for some $\left.p \in(1, \infty)\right)$. Here, the convergence of the principal value limit holds pointwise a.e. and in $L^{p}$, for $f \in L^{p}, 1<p<\infty$. We shall not explore pointwise convergence further in the present survey, but see, e.g., [86], Chapters II-III, and [87], Chapter I, Section 7.

In higher dimensions, the operators analogous to $H$ are the Riesz transforms

$$
R_{j} f(x):=p \cdot v \cdot \frac{2}{\sigma_{n}} \int_{\mathbb{R}^{n}} \frac{x_{j}-y_{j}}{|x-y|^{n+1}} f(y) d y, \quad j=1,2, \ldots, n,
$$

where $\sigma_{n}$ is the volume of the unit $n$-sphere in $\mathbb{R}^{n+1}$. The Riesz transforms relate the tangential and normal derivatives of a harmonic function $u$ in the half-space $\mathbb{R}_{+}^{n+1}:=$ $\left\{(x, t) \in \mathbb{R}^{n} \times(0, \infty)\right\}$, via the formula $R_{j}\left(\partial_{t} u(\cdot, t)\right)=\partial_{x_{j}} u(\cdot, t)$, assuming, say, $u(\cdot, t) \in L^{p}\left(\mathbb{R}^{n}\right)$. They also arise naturally in the study of $W^{2, p}$ regularity of solutions of Poisson's equation $\Delta u=f$ in $\mathbb{R}^{n}$ (see [86], Chapter III).

We observe that the two examples (1.5) and (1.6) are both of convolution type, i.e., $K(x, y)=K(x-y)$. We shall discuss convolution operators further in Section 3.1.

We now mention some examples that are not of convolution type. The Calderón Commutators are the operators

$$
C_{A}^{k} f(x):=p \cdot v \cdot \frac{i}{2 \pi} \int_{\mathbb{R}}\left(\frac{A(x)-A(y)}{x-y}\right)^{k} \frac{1}{x-y} f(y) d y
$$


where $A$ is a Lipschitz function. Observe that, up to normalization, the case $k=0$ is the Hilbert transform, and that at least formally, $C_{A}^{1}$ is a commutator:

$$
C_{A}^{1} f=\frac{i}{2}\left[\frac{d}{d x} H, A\right] f:=\frac{i}{2}\left(\frac{d}{d x}(H A f)-A \frac{d}{d x}(H f)\right)
$$

while $C_{A}^{k}$ is a higher commutator $(k=2,3, \ldots)$ :

$$
C_{A}^{k} f=\frac{i}{k ! 2}\left[\ldots\left[\left[\frac{d^{k}}{d x^{k}} H, A\right] A\right] \ldots A\right] f
$$

The operator $C_{A}^{1}$ (and its higher dimensional analogues) arose in Calderón's construction of an algebra of SIOs suitable for the treatment of partial differential operators with merely Lipschitz coefficients, thus, a sort of pseudo-differential calculus which, in contrast to the classical pseudo-differential calculus, was applicable to operators with rather minimally smooth coefficients [26].

Moreover, the family of operators $C_{A}^{k}$ arise in the power series expansion of the operator

$$
\mathfrak{C}_{A} f(x):=p \cdot v \cdot \frac{i}{2 \pi} \int_{\mathbb{R}} \frac{1}{x-y+i(A(x)-A(y))} f(y) d y,
$$

namely

$$
\mathfrak{C}_{A}=\sum_{k=0}^{\infty}(-i)^{k} C_{A}^{k}
$$

at least when $\left\|A^{\prime}\right\|_{\infty}<1$. In turn, the operator $\mathfrak{C}_{A}$ arises when writing the parametric representation of the Cauchy singular integral operator on a Lipschitz graph. More precisely, set

$$
\mathcal{C}_{\gamma} g(z):=p \cdot v \cdot \frac{i}{2 \pi} \int_{\gamma} \frac{1}{z-v} g(v) d v .
$$

If $\gamma$ is a Lipschitz curve in the complex plane $\mathbb{C}$ parametrized by $z=x+i A(x)$, then

$$
\begin{aligned}
\mathfrak{C}_{A} f(x) & =\mathcal{C}_{\gamma} g(x+i A(x)), \quad \text { where } \\
f(y) & :=\left(1+i A^{\prime}(y)\right) g(y+i A(y)) .
\end{aligned}
$$

Of course, the role of the Cauchy integral in complex function theory is well known. We observe that, for $A$ Lipschitz, the kernels $K(x, y)=(A(x)-A(y))^{k} /(x-y)^{k+1}$ and $K(x, y)=(x-y+i(A(x)-A(y)))^{-1}$, corresponding to the operators $C_{A}^{k}$ and $\mathfrak{C}_{A}$ respectively, satisfy the Calderón-Zygmund kernel conditions (1.2) and (1.3), as the reader may readily verify. 
Calderón's lecture at the International Congress of Mathematicians in Helsinki in 1978 contains a clear account of the state of the art at that time concerning commutators, Cauchy integrals on Lipschitz curves and applications [28].

Let us note that for all of the operators (1.5), (1.6), (1.7) and (1.9), the kernel $K(x, y)$ is anti-symmetric, i.e.,

$$
K(x, y)=-K(y, x) .
$$

For all anti-symmetric kernels which satisfy the pointwise kernel bound (1.2) $|K(x, y)| \leq C|x-y|^{-n}$, the associated principal value operator is always well defined, at least in the sense of distributions. Indeed, in that case, one may extend the representation (1.1) as follows. For all $\varphi, \psi \in C_{0}^{\infty}\left(\mathbb{R}^{n}\right)$ (with supports that are not necessarily disjoint), the principal value

$$
\langle T \varphi, \psi\rangle:=\lim _{\varepsilon \rightarrow 0} \iint_{|x-y|>\varepsilon} \psi(x) K(x, y) \varphi(y) d y d x
$$

exists. Moreover it satisfies the Weak Boundedness Property (WBP), i.e. there exists $C=C(K, n)$ such that

$$
|\langle T \varphi, \psi\rangle| \leq C R^{n}\left\{\|\varphi\|_{\infty}+R\|\nabla \varphi\|_{\infty}\right\}\left\{\|\psi\|_{\infty}+R\|\nabla \psi\|_{\infty}\right\}
$$

for all $R>0$ and $x \in \mathbb{R}^{n}$, and all test functions $\varphi, \psi$ supported in the ball

$$
B(x, R):=\left\{y \in \mathbb{R}^{n}:|x-y|<R\right\} .
$$

Indeed, to verify (1.12) and (1.13), we use (1.11) and then a re-labelling of the variables to write

$$
\begin{aligned}
& \left\langle T_{\varepsilon} \varphi, \psi\right\rangle:=\iint_{|x-y|>\varepsilon} \psi(x) K(x, y) \varphi(y) d y d x \\
& =-\iint_{|x-y|>\varepsilon} \psi(x) K(y, x) \varphi(y) d y d x=-\iint_{|x-y|>\varepsilon} \psi(y) K(x, y) \varphi(x) d x d y,
\end{aligned}
$$

and thus

$$
\left\langle T_{\varepsilon} \varphi, \psi\right\rangle=\frac{1}{2} \iint_{|x-y|>\varepsilon} K(x, y)(\psi(x) \varphi(y)-\psi(y) \varphi(x)) d y d x .
$$

Written this way, the integrand is only weakly singular, in the sense that the kernel bound (1.2) $|K(x, y)| \leq C|x-y|^{-n}$ has been improved to

$$
\begin{aligned}
|K(x, y)(\psi(x) \varphi(y)-\psi(y) \varphi(x))| & \leq|K(x, y) \psi(x)(\varphi(y)-\varphi(x))+\varphi(x)(\psi(x)-\psi(y))| \\
& \leq C^{\prime}|x-y|^{-n+1}\left\{\|\psi\|_{\infty}\|\nabla \varphi\|_{\infty}+\|\varphi\|_{\infty}\|\nabla \psi\|_{\infty}\right\}
\end{aligned}
$$


from which it is easy to deduce convergence of the limit in (1.12), along with the bound (1.13).

We remark that the weak boundedness property (1.13) holds for any $L^{2}$ bounded operator $T$, with $C \approx\|T\|_{o p}$ (by Cauchy-Schwarz), and for an SIO should be viewed as expressing some cancellation in the operator $T$.

We remark also that there are $L^{2}$ bounded singular integral operators $T$ which do not satisfy (1.12). Indeed some care is needed, for in our definition of an SIO, nothing is said about the behaviour of $K(x, y)$ when $x=y$. So the $L^{2}$ bounded operator $T=I$ is associated to the kernel $K(x, y) \equiv 0$, as also is the unbounded operator $T=\frac{d}{d x}$, though the latter does not satisfy WBP.

\subsection{Square functions}

Closely related to SIOs are the square functions. These can arise in the analysis of SIOs, and in turn, may be viewed as singular integrals with kernels taking their values in a Hilbert space. They are also of interest in their own right, especially in applications to partial differential equations. We describe one particular set-up, but note that there are several others of interest, as e.g. in [85].

Following Christ and Journé [33] and Christ [32], we say that a family of kernels $\left\{\psi_{t}(x, y)\right\}_{t \in(0, \infty)}$, is a standard Littlewood-Paley family if, for some exponent $\alpha>0$ and $C<\infty$, we have

$$
\begin{array}{cc}
\left|\psi_{t}(x, y)\right| \leq C \frac{t^{\alpha}}{(t+|x-y|)^{n+\alpha}} \quad \text { and } \\
\left|\psi_{t}(x, y+h)-\psi_{t}(x, y)\right| \leq C \frac{|h|^{\alpha}}{(t+|x-y|)^{n+\alpha}}, \quad|h| \leq t,
\end{array}
$$

for all $x, y \in \mathbb{R}^{n}$ and $t>0$. Often, one may also have local Hölder continuity in the $x$ variable:

$$
\left|\psi_{t}(x+h, y)-\psi_{t}(x, y)\right| \leq C \frac{|h|^{\alpha}}{(t+|x-y|)^{n+\alpha}}, \quad|h| \leq t .
$$

For any family $\left\{\psi_{t}\right\}$ which satisfies (1.14), one can show that

$$
\left|\int_{\mathbb{R}^{n}} \psi_{t}(x, y) f(y) d y\right| \lesssim M f(x)
$$

where $M$ denotes the Hardy-Littlewood maximal operator which is well known to be bounded on every $L^{p}, 1<p \leq \infty$. See (1.30). Thus, in particular, the linear operators $\Theta_{t}$ defined by

$$
\Theta_{t} f(x):=\int_{\mathbb{R}^{n}} \psi_{t}(x, y) f(y) d y
$$


are uniformly bounded in $L^{\infty}\left(\mathbb{R}^{n}\right)$ with

$$
\left\|\Theta_{t} b\right\|_{\infty} \leq C_{n, \alpha}\|b\|_{\infty}
$$

for all $b \in L^{\infty}\left(\mathbb{R}^{n}\right)$ and all $t>0$, and are uniformly bounded in $L^{2}\left(\mathbb{R}^{n}\right)$ with

$$
\left\|\Theta_{t} f\right\|_{2} \leq C_{n, \alpha}\|f\|_{2}
$$

for all $f \in L^{2}\left(\mathbb{R}^{n}\right)$ and all $t>0$.

Alternatively, one may observe that (1.14) implies uniform $L^{1}$ bounds

$$
\begin{aligned}
& \max \left\{\sup _{y \in \mathbb{R}^{n}} \int_{\mathbb{R}^{n}}\left|\psi_{t}(x, y)\right| d x, \sup _{x \in \mathbb{R}^{n}} \int_{\mathbb{R}^{n}}\left|\psi_{t}(x, y)\right| d y\right\} \\
& \leq C \int_{\mathbb{R}^{n}} \frac{t^{\alpha}}{(t+|z|)^{n+\alpha}} d z=C \int_{\mathbb{R}^{n}} \frac{1}{(1+|z|)^{n+\alpha}} d z=: C_{n, \alpha},
\end{aligned}
$$

whence (1.17) follows directly, and then (1.18) follows either by duality and interpolation, or else as a consequence of the Schur estimate (1.32) below.

We now define two square function operators:

$$
\begin{aligned}
G_{\psi} f(x) & :=\left(\int_{0}^{\infty}\left|\Theta_{t} f(x)\right|^{2} \frac{d t}{t}\right)^{1 / 2} \text { and } \\
S_{\psi} f(x) & :=\left(\iint_{\Gamma(x)}\left|\Theta_{t} f(y)\right|^{2} \frac{d y d t}{t^{n+1}}\right)^{1 / 2},
\end{aligned}
$$

where $\Gamma(x):=\left\{(y, t) \in \mathbb{R}_{+}^{n+1}:|x-y|<t\right\}$ is the standard cone with vertex at $x$. For obvious reasons, $G_{\psi}$ and $S_{\psi}$ are typically referred to as vertical and conical square functions, respectively. We remark that they have equivalent $L^{2}$ norms:

$$
\begin{aligned}
\left\|S_{\psi} f\right\|_{2}^{2} & =\int_{\mathbb{R}^{n}} \int_{0}^{\infty} \int_{|x-y|<t}\left|\Theta_{t} f(y)\right|^{2} d y \frac{d t}{t^{n+1}} d x \\
& =\int_{0}^{\infty} \int_{\mathbb{R}^{n}}\left(\frac{1}{t^{n}} \int_{|x-y|<t} d x\right)\left|\Theta_{t} f(y)\right|^{2} d y \frac{d t}{t}=\omega_{n}\left\|G_{\psi} f\right\|_{2}^{2}
\end{aligned}
$$

where $\omega_{n}$ is the volume of the unit ball in $\mathbb{R}^{n}$. The choice of aperture 1 in the cone defining $S_{\psi}$ is merely a normalization, and one could just as well integrate over the cone with any other fixed aperture $\beta>0$, i.e., $\Gamma_{\beta}(x):=\left\{(y, t) \in \mathbb{R}_{+}^{n+1}:|x-y|<\beta t\right\}$.

The prototypical example is

$$
\psi_{t}(x, y)=\psi_{t}(x-y)=t \frac{\partial}{\partial t} p_{t}(x-y)
$$


where

$$
p_{t}(x)=\frac{2 t}{\sigma_{n}\left(t^{2}+|x|^{2}\right)^{(n+1) / 2}}
$$

is the classical Poisson kernel for the Laplacian in the half-space $\mathbb{R}_{+}^{n+1}$, with $\sigma_{n}$ denoting the volume of the unit $n$-sphere in $\mathbb{R}^{n+1}$. Thus,

$$
\Theta_{t} f=\psi_{t} * f=t \frac{\partial}{\partial t} \mathcal{P}_{t} f
$$

where $\mathcal{P}_{t}:=e^{-t \sqrt{-\Delta}}$ is the Poisson semigroup, so that the Fourier transform is

$$
\widehat{\Theta_{t} f}(\xi)=\widehat{\psi}(t \xi) f(\xi)=-2 \pi t|\xi| e^{-2 \pi t|\xi|} f(\xi)
$$

(See Section 3.1.) Note that in this case the operator $\Theta_{t}$ is of convolution type. Not surprisingly, the corresponding square function operators $G_{\psi}$ and $S_{\psi}$ play a fundamental role in the theory of harmonic functions in $\mathbb{R}_{+}^{n+1}$.

A general class of convolution examples is provided by choosing $\psi \in C_{0}^{\infty}\left(\mathbb{R}^{n}\right)$ (or more generally, in the Schwarz class $\mathcal{S}$ ), with $\int_{\mathbb{R}^{n}} \psi(x) d x=0$, and setting

$$
\psi_{t}(x):=\frac{1}{t^{n}} \psi\left(\frac{x}{t}\right), \quad Q_{t} f:=\psi_{t} * f
$$

It is an easy exercise to verify that the functions $\psi_{t}(x, y):=\psi_{t}(x-y)$ form a standard Littlewood-Paley family with $\alpha=1$. In this situation, it is the usual notational convention to use $Q_{t}$ in place of $\Theta_{t}$, thus $Q_{t} f:=\psi_{t} * f, G_{\psi} f=\left(\int_{0}^{\infty}\left|Q_{t} f\right|^{2} d t / t\right)^{1 / 2}$, and so on.

An important class of non-convolution examples arises in the theory of divergence form elliptic operators. Let $A$ be an $n \times n$ matrix with bounded measurable entries

$$
A_{j k}: \mathbb{R}^{n} \longrightarrow \mathbb{C}, \quad j=1, \ldots, n, \quad k=1, \ldots, n,
$$

satisfying the ellipticity condition

$$
\lambda|\xi|^{2} \leq \Re e A(x) \xi \cdot \bar{\xi} \text { and }|A(x) \xi \cdot \bar{\zeta}| \leq \Lambda|\xi||\zeta|, \quad \forall \xi, \zeta \in \mathbb{C}^{n}, x \in \mathbb{R}^{n} \text { (a.e.) }
$$

for some constants $0<\lambda \leq \Lambda<\infty$. Let $L$ denote the second order divergence form operator defined by

$$
L f:=-\operatorname{div}(A \nabla f)=-\sum_{1 \leq j, k \leq n} \frac{\partial}{\partial x_{j}}\left(A_{j k} \frac{\partial f}{\partial x_{k}}\right),
$$


which we interpret in the usual weak sense via a sesquilinear form, and let $p_{t}^{L}(x, y)$ and $\mathcal{P}_{t}^{L}:=e^{-t \sqrt{L}}$ be the associated Poisson kernel and Poisson semigroup, respectively. Generalizing (1.23), we then set

$$
\psi_{t}(x, y):=t \frac{\partial}{\partial t} p_{t}^{L}(x-y), \quad \Theta_{t}:=t \frac{\partial}{\partial t} \mathcal{P}_{t}^{L}
$$

If the coefficient matrix $A$ has real entries, then this kernel $\psi_{t}$ satisfies the bounds (1.14), (1.15) and (1.16) by the classical De Giorgi/Nash regularity theory [43,78]. The De Giorgi/Nash estimates remain true for complex coefficients when $n=2$ [16], or when $A$ is sufficiently close (in $L^{\infty}$ ) to a real matrix [1,18]. On the other hand, in general, $\psi_{t}(x, y)$ need not satisfy the pointwise bounds (1.14), (1.15) nor (1.16), yet even then it is still possible to develop some aspects of the theory. We refer the reader to [3] and to [11] for a discussion of the latter situation.

\subsection{Notation}

The Lebesgue measure of a measurable subset $S \subset \mathbb{R}^{n}$ is denoted by $|S|$, while its indicator function $1_{S}$ is defined by $1_{S}(x)=1$ if $x \in S$ and $1_{S}(x)=0$ if $x \in \mathbb{R}^{n} \backslash S$.

For each cube $Q \subset \mathbb{R}^{n}$, the mean value of a function $f$ over $Q$ is defined to be

$$
[f]_{Q}:=|Q|^{-1} \int_{Q} f(x) d x,
$$

while $\ell(Q)$ denotes its side length, and $\kappa Q$ denotes the concentric dilate of $Q$ by a factor of $\kappa>0$. Our cubes always have sides parallel to the coordinate axes.

For each integer $N$, the symbol $\mathbb{D}(N)$ denotes the collection of all dyadic cubes in $\mathbb{R}^{n}$ with $\ell(Q)=2^{N}$, that is, the collection of cubes $2^{N} \tilde{k}+\left(0,2^{N}\right]^{n}$ with $\tilde{k} \in \mathbb{Z}^{n}$, and $\mathbb{D}:=\bigcup_{N \in \mathbb{Z}} \mathbb{D}(N)$.

For each $x \in \mathbb{R}^{n}$ and $r>0, B(x, r)$ denotes the open ball with centre $x$ and radius $r$.

The Hardy-Littlewood maximal function of a measurable function $f$ defined on $\mathbb{R}^{n}$ is $M f(x):=\sup _{r>0}|B(x, r)|^{-1} \int_{B(x, r)} f(y) d y$. We use the well-known result that, when $1<p \leq \infty$, there exists $C_{n, p}<\infty$ such that

$$
\|M f\|_{p} \leq C_{n, p}\|f\|_{p}
$$

for all $f \in L^{p}\left(\mathbb{R}^{n}\right)$. (This is the first theorem in [86].)

We use the notation $X \lesssim Y$ to mean that there exists a constant $C>0$ such that $X \leq C Y$. The notation $X \approx Y$ means that $X \lesssim Y$ and $Y \lesssim X$. The value of $C$ varies from one usage to the next.

For later use, we note Chebychev's inequality with exponent $p \in[1, \infty)$ : For each $g \in L^{p}\left(\mathbb{R}^{n}\right)$ and $\lambda>0$,

$$
\lambda^{p}\left|\left\{x \in \mathbb{R}^{n} ;|g(x)|>\lambda\right\}\right| \leq\|g\|_{p}^{p},
$$


which is easily verified, as is the Schur estimate for integral operators $S f(x)=$ $\int_{\mathbb{R}^{n}} K(x, y) f(y) d y$ with weakly singular kernels:

$$
\|S f\|_{2} \leq\left\{\sup _{y \in \mathbb{R}^{n}} \int_{\mathbb{R}^{n}}|K(x, y)| d x\right\}^{1 / 2}\left\{\sup _{x \in \mathbb{R}^{n}} \int_{\mathbb{R}^{n}}|K(x, y)| d y\right\}^{1 / 2}\|f\|_{2} .
$$

The theory of SIOs deals with integral operators with kernels which are not absolutely integrable in this sense, but whose boundedness depends on some cancelation properties of the kernel.

\section{$2 L^{p}$ and endpoint theory of SIOs and square functions}

In this section, we discuss $L^{p}$ and endpoint bounds that are satisfied by SIOs and square functions, assuming boundedness on $L^{2}$. The fundamental result is that of Calderón and Zygmund [40].

Theorem 2.1 (Calderón-Zygmund Theorem) [40]. Let T be an SIO associated to a Calderón-Zygmund kernel $K(x, y)$ satisfying the standard bounds (1.2) and (1.3). Suppose also that $T$ extends to a bounded linear operator on $L^{2}\left(\mathbb{R}^{n}\right)$, i.e., that for all $f, g \in C_{0}^{\infty}\left(\mathbb{R}^{n}\right)$, we have

$$
|\langle T f, g\rangle| \leq C\|f\|_{2}\|g\|_{2}
$$

Then $T$ extends to a bounded operator on $L^{p}\left(\mathbb{R}^{n}\right), 1<p<\infty$.

To be historically accurate, we should observe that the original paper [40] treated only the convolution case, but the argument there extends essentially verbatim to the general setting described above. Let us now give the proof, following [40]. We begin with a fundamental stopping time lemma, in which an $L^{1}$ function $f$ is decomposed into a good part $g$ and a bad part $b$.

Lemma 2.2 (Calderón-Zygmund decomposition). Suppose that $f \in L^{1}\left(\mathbb{R}^{n}\right)$, and let $\lambda>0$. Then there is a family $\mathcal{F}:=\left\{Q_{j}\right\}_{j=1}^{\infty}$ of non-overlapping dyadic cubes, and a decomposition $f=b+g$ such that

(1) $\lambda \leq\left|Q_{j}\right|^{-1} \int_{Q_{j}}|f(x)| d x<2^{n} \lambda$, for every $Q_{j} \in \mathcal{F}$;

(2) $\sum_{j}\left|Q_{j}\right| \leq \lambda^{-1}\|f\|_{1}$;

(3) $b=\sum_{j} b_{Q_{j}}$, with $\operatorname{supp}\left(b_{Q_{j}}\right) \subset Q_{j}$, and $\int b_{Q_{j}}=0, \forall Q_{j} \in \mathcal{F}$;

(4) $g \in L^{1}\left(\mathbb{R}^{n}\right) \cap L^{2}\left(\mathbb{R}^{n}\right)$, with $\|g\|_{1} \leq\|f\|_{1}$ and $\|g\|_{2}^{2} \leq C_{n} \lambda\|f\|_{1}$.

Proof of Lemma 2.2 We start with an initial grid $\mathbb{D}(N)$ of dyadic cubes of side length $2^{N}$, chosen so large that for $Q \in \mathbb{D}(N)$, we have

$$
\frac{1}{|Q|} \int_{Q}|f(x)| d x \leq \frac{1}{|Q|}\|f\|_{1}<\lambda .
$$


We then subdivide each $Q$ in $\mathbb{D}(N)$ dyadically, stopping the first time we reach a sub-cube $Q^{\prime} \subset Q$ for which

$$
\frac{1}{\left|Q^{\prime}\right|} \int_{Q^{\prime}}|f(x)| d x \geq \lambda
$$

The family $\mathcal{F}$ is simply the collection of cubes that are maximal with respect to the property (2.3). The left hand inequality in (1) then holds by definition, while the right hand bound follows from the maximality of $Q_{j} \in \mathcal{F}$. In turn, (2) follows directly from the left hand estimate in (1), since the cubes $Q_{j}$ are non-overlapping (by maximality).

To establish (3) and (4), we set $E:=\cup_{j} Q_{j}$, and define

$$
g:=f 1_{\mathbb{R}^{n} \backslash E}+\sum_{j} 1_{Q_{j}} \frac{1}{\left|Q_{j}\right|} \int_{Q_{j}} f(x) d x, \quad b:=f-g .
$$

Then (3) holds by definition, with

$$
b_{Q_{j}}=\left(f-[f]_{Q_{j}}\right) 1_{Q_{j}},
$$

recalling that $[f]_{Q_{j}}$ denotes the mean value of $f$ over $Q_{j}$ (1.29).

To prove (4), we observe first that the claimed $L^{1}$ bound for $g$ is trivial, while the $L^{2}$ bound may be obtained from the $L^{1}$ bound and the fact that $\|g\|_{\infty} \leq 2^{n} \lambda$. In turn, the latter estimate follows directly from Lebesgue's differentiation theorem (to control $g$ on $\mathbb{R}^{n} \backslash E$ ), and the right hand inequality in (1).

Proof of Theorem 2.1 We shall obtain this result by interpolation and duality, after first establishing the weak-type $(1,1)$ bound:

$$
\sup _{\lambda>0}\left(\lambda\left|\left\{x \in \mathbb{R}^{n}:|T f(x)|>\lambda\right\}\right|\right) \leq C\|f\|_{1},
$$

where $C$ depends only on dimension, the kernel bounds (1.2) and (1.3), and the $L^{2} \rightarrow$ $L^{2}$ operator norm of $T$. It is enough to prove (2.4) for $f \in L^{2} \cap L^{1}$, as one may then extend by continuity to all of $L^{1}$. To this end, we fix $\lambda>0$, and write $f=b+g$ as in Lemma 2.2. Then

$\left|\left\{x \in \mathbb{R}^{n}:|T f(x)|>\lambda\right\}\right| \leq\left|\left\{x \in \mathbb{R}^{n}:|T b(x)|>\lambda / 2\right\}\right|+\left|\left\{x \in \mathbb{R}^{n}:|\operatorname{Tg}(x)|>\lambda / 2\right\}\right|$.

The desired bound for $T g$ follows directly from Chebychev's inequality with exponent 2 and the $L^{2}$ bound for $g$ in Lemma 2.2:

$$
\left|\left\{x ;|T g(x)|>\frac{\lambda}{2}\right\}\right| \leq \frac{4}{\lambda^{2}}\|T g\|_{2}^{2} \leq \frac{4 C_{n}}{\lambda}\|T\|_{o p}^{2}\|f\|_{1} .
$$


To handle $T b$, we set $E_{*}:=\cup_{j}\left(5 Q_{j}\right)$. Then

$$
\begin{aligned}
\left|\left\{x \in \mathbb{R}^{n}:|T b(x)|>\lambda / 2\right\}\right| & \leq \sum_{j}\left|5 Q_{j}\right|+\left|\left\{x \in \mathbb{R}^{n} \backslash E_{*}:|T b(x)|>\lambda / 2\right\}\right| \\
& =: I+I I .
\end{aligned}
$$

By Lemma 2.2 (2), we have that $I \leq 5^{n} \lambda^{-1}\|f\|_{1}$, as desired.

It now remains to treat term $I I$. We proceed as follows. First, by our qualitative assumption that $f \in L^{2} \cap L^{1}$, we have in particular that the sum in Lemma 2.2 converges in $L^{2}$. Since $T: L^{2} \rightarrow L^{2}$, it therefore follows that

$$
|T b(x)| \leq \sum_{j}\left|T b_{Q_{j}}(x)\right| \text { a.e. }
$$

so that by Chebychev's inequality we have

$$
I I \leq \frac{2}{\lambda} \sum_{j} \int_{\mathbb{R}^{n} \backslash E_{*}}\left|T b_{Q_{j}}(x)\right| d x .
$$

Next, we let $y_{j}$ denote the center of $Q_{j}$, and note that by (1.4), the fact that $b_{Q_{j}}$ has mean value 0 , and (1.3), we have

$$
\begin{aligned}
\left|T b_{Q_{j}}(x)\right| & =\left|\int K(x, y) b_{Q_{j}}(y) d y\right|=\left|\int\left(K(x, y)-K\left(x, y_{j}\right)\right) b_{Q_{j}}(y) d y\right| \\
& \leq C \frac{\ell\left(Q_{j}\right)^{\alpha}}{\left(\ell\left(Q_{j}\right)+\left|x-y_{j}\right|\right)^{n+\alpha}} \int\left|b_{Q_{j}}(y)\right| d y, \quad \forall x \in \mathbb{R}^{n} \backslash 5 Q_{j},
\end{aligned}
$$

where $\ell(Q)$ denotes the side length of $Q$. Combining (2.5) and (2.6), we obtain

$$
I I \leq \frac{C_{n, \alpha}}{\lambda} \sum_{j} \int\left|b_{Q_{j}}(y)\right| d y=\frac{C_{n, \alpha}}{\lambda} \int|b(y)| d y \leq 2 \frac{C_{n, \alpha}}{\lambda}\|f\|_{1},
$$

thus completing the proof of (2.4).

By the Marcinkiewicz interpolation theorem, as presented e.g. in Theorem 5, Ch. I of [86], it follows from the $L^{2}$ bound for $T$ and the weak-type $(1,1)$ bound (2.4), that $T$ is bounded in $L^{p}$ for all $p \in(1,2]$. By the symmetry of the kernel conditions (1.2) and (1.3), the same is true for its transpose ${ }^{t} T$. So, by duality, $T$ is bounded in $L^{p}$ for all $p \in[2, \infty)$, and the proof is complete.

We shall not discuss $L^{p}$ theory for square functions explicitly, but let us simply note that, assuming (1.14)-(1.16), the square function operators $G_{\psi}$ and $S_{\psi}$ may be viewed as SIOs with standard kernels taking values in an appropriate Hilbert space, and thus bounded on $L^{p}$, given $L^{2}$ boundedness. For example, in the case of $G_{\psi}$, we set

$$
K(x, y)=\{K(x, y)(t)\}_{t}:=\left\{\psi_{t}(x, y)\right\}_{t},
$$


and observe that $K(x, y)$ satisfies the kernel bounds (1.2), (1.3), if the modulus $|\cdot|$ is replaced by the Hilbert space norm

$$
\|h\|_{\mathcal{H}}:=\left(\int_{0}^{\infty}|h(t)|^{2} \frac{d t}{t}\right)^{1 / 2} .
$$

We leave this observation as an exercise for the reader. In this context, the proof of the Calderón-Zygmund Theorem 2.1 carries over mutatis mutandi.

It is worth emphasizing that the $L^{p}$ bounds in Theorem 2.1 were obtained by interpolating between the assumed $L^{2}$ estimate and an endpoint estimate, in this case a weak-type $(1,1)$ bound. However, there is another type of endpoint estimate which may also serve via interpolation to obtain $L^{p}$ bounds, namely, the fact that an $L^{2}$ bounded SIO maps $L^{\infty}\left(\mathbb{R}^{n}\right)$ into $B M O\left(\mathbb{R}^{n}\right)$, the space of functions of bounded mean oscillation.

We recall that $B M O\left(\mathbb{R}^{n}\right)$ is the Banach space of locally integrable functions modulo constants for which the norm

$$
\|b\|_{*}=\sup |Q|^{-1} \int_{Q}\left|b(x)-[b]_{Q}\right| d x
$$

is finite, where the supremum runs over all cubes $Q \subset \mathbb{R}^{n}$ with sides parallel to the co-ordinate axes (though balls would work just as well). The fundamental result about $B M O$ is the John-Nirenberg Theorem [67], which implies in particular that, when $1 \leq p<\infty$,

$$
\|b\|_{*} \approx \sup \left(|Q|^{-1} \int_{Q}\left|b(x)-[b]_{Q}\right|^{p} d x\right)^{1 / p},
$$

where the implicit constants depend only on $p$ and dimension. We also note the elementary fact that the mean value $[b]_{Q}$ is essentially the optimal constant. More precisely,

$$
\frac{1}{2}\|b\|_{*} \leq \operatorname{supinf}|Q|^{-1} \int_{Q}\left|b(x)-c_{Q}\right| d x \leq\|b\|_{*},
$$

where the infimum runs over all constants $c_{Q}$, and the supremum again runs over all cubes $Q \subset \mathbb{R}^{n}$.

The following result was obtained independently by Peetre [81], Spanne [83] and Stein [84]:

Theorem 2.9 (Peetre-Spanne-Stein Theorem). Let $T$ be an $L^{2}$ bounded SIO, associated to a standard Calderón-Zygmund kernel $K(x, y)$ (cf. (1.2), (1.3).) Then the mapping $T: L^{\infty}\left(\mathbb{R}^{n}\right) \rightarrow B M O\left(\mathbb{R}^{n}\right)$ is bounded.

Proof Fix $Q$, and let $f \in L^{\infty}$. We write $f=f_{1}+f_{2}$, where $f_{1}:=f 1_{5 Q}$. Let $x_{Q}$ denote the center of $Q$, and set $c_{Q}:=T f_{2}\left(x_{Q}\right)$. We then have

$$
\int_{Q}\left|T f(x)-c_{Q}\right| d x \leq \int_{Q}\left|T f_{1}(x)\right| d x+\int_{Q}\left|T f_{2}(x)-c_{Q}\right| d x=: I+I I .
$$


By Cauchy-Schwarz and the $L^{2}$ boundedness of $T$, we have that

$$
I \leq\|T\|_{o p}|Q|^{1 / 2}\left\|f_{1}\right\|_{2} \leq 5^{n / 2}\|T\|_{o p}|Q|\|f\|_{\infty} .
$$

Also, by (1.3) we have, for $x \in Q$ :

$$
\begin{aligned}
\left|T f_{2}(x)-c_{Q}\right| & =\left|\int_{\mathbb{R}^{n} \backslash 5 Q}\left(K(x, y)-K\left(x_{Q}, y\right)\right) f(y) d y\right| \\
& \leq C\|f\|_{\infty} \int_{\mathbb{R}^{n}} \frac{\ell(Q)^{\alpha}}{\left(\ell(Q)+\left|x_{Q}-y\right|\right)^{n+\alpha}} d y=C_{n, \alpha}\|f\|_{\infty},
\end{aligned}
$$

whence $I I \leq C\|f\|_{\infty}|Q|$. The conclusion of the theorem then follows by (2.8).

Note that, implicitly, $T f$ is only defined modulo constants for general $f \in L^{\infty}$.

A few remarks are in order. Suppose that $T$ is an $L^{2}$ bounded SIO. By the symmetry of the kernel conditions (1.2) and (1.3), the same is true for its transpose ${ }^{t} T$. Then by Theorem 2.9, both $T$ and ${ }^{t} T$ map $L^{\infty}$ into $B M O$, whence by Fefferman's duality theorem [51], $T$ also maps the Hardy space $H^{1}\left(\mathbb{R}^{n}\right)$ into $L^{1}$. By the Fefferman-Stein interpolation theorem [51], we then have that $T: L^{p} \rightarrow L^{p}, 1<p<\infty$, thus providing an alternative proof of Theorem 2.1.

Moreover, the same line of reasoning leads to a more subtle observation, which in turn serves to clarify the nature of the results in the next section. Notice that if we start with the hypothesis that $T$ and ${ }^{t} T$ both map $L^{\infty}$ into $B M O$, then the same duality and interpolation arguments yield that $T$ is bounded on $L^{2}$. The $L^{2}$ boundedness criteria that we shall discuss in Section 3 state that, rather than testing $T$ and ${ }^{t} T$ on all of $L^{\infty}$, it is enough to verify that each of them maps one particular function in $L^{\infty}$ into BMO.

Finally, Theorem 2.9, and also the Calderón Zygmund Theorem 2.1, say that given $L^{2}$ boundedness of an SIO associated to a standard kernel, one then automatically knows $L^{p}$ and endpoint estimates for the operator. Thus, the question of $L^{2}$ boundedness is paramount. We remark that one can also obtain weighted $L^{p}$ estimates. See, e.g. [53]. The weighted theory is also of considerable interest, and has, in fact, enjoyed a renaissance of late. However, we shall not touch on this subject in the present survey.

\section{$3 L^{2}$ boundedness criteria}

\subsection{The convolution case}

The theory of convolution integral operators is closely related to Fourier theory. Suppose $\psi \in L^{1}\left(\mathbb{R}^{n}\right)$. Its Fourier transform $\hat{\psi}$, defined by

$$
\hat{\psi}(\xi)=\int_{\mathbb{R}^{n}} e^{-2 \pi i \xi \cdot x} \psi(x) d x
$$

is a continuous function which satisfies $\|\widehat{\psi}\|_{\infty} \leq\|\psi\|_{1}$ and the Plancherel identity $\|\widehat{\psi}\|_{2}=\|\psi\|_{2}$ provided also that $\psi \in L^{2}\left(\mathbb{R}^{n}\right)$. The transform of convolution is mul- 
tiplication, in the sense that $\widehat{\psi * f}(\xi)=\widehat{\psi}(\xi) \widehat{f}(\xi)$ for all $\xi \in \mathbb{R}^{n}$ when $f \in L^{1}\left(\mathbb{R}^{n}\right)$. Hence

$$
\|\psi * f\|_{2}=\|\widehat{\psi} \widehat{f}\|_{2} \leq\|\widehat{\psi}\|_{\infty}\|\widehat{f}\|_{2} \leq\|\psi\|_{1}\|f\|_{2}
$$

for all $\psi \in L^{1}\left(\mathbb{R}^{n}\right)$ and $f \in L^{1} \cap L^{2}\left(\mathbb{R}^{n}\right)$.

The question of $L^{2}$ boundedness of a convolution SIO with kernel $K(x, y)=$ $K(x-y)$ is straightforward: by Plancherel's Theorem, it is sufficient to verify that the associated Fourier multiplier

$$
m(\xi):=\lim _{\varepsilon \rightarrow 0} \int_{\varepsilon<|x|<\varepsilon^{-1}} e^{-2 \pi i \xi \cdot x} K(x) d x
$$

belongs to $L^{\infty}$. In turn, it is not hard to establish the boundedness of $m$, given the standard kernel estimates (1.2), (1.3) and sufficient cancellation, say that $K$ has mean value 0 on every annulus $0<a<|x|<b<\infty$. Clearly, the latter cancellation condition holds for any odd kernel $K$, for example as one encounters in the Hilbert and Riesz transforms. In this case, we have formally that $T f=K * f$, or $\widehat{T f}(\xi)=m(\xi) \widehat{f}(\xi)$, so $T$ can be written in terms of the bounded functional calculus of commuting self-adjoint operators:

$$
(T f)(x)=m\left(\frac{1}{2 \pi i}\left(\frac{\partial}{\partial x_{1}}, \frac{\partial}{\partial x_{2}}, \ldots, \frac{\partial}{\partial x_{n}}\right)\right) f(x)
$$

with $\|T\|_{o p}=\|m\|_{\infty}$ as $\|T f\|_{2}=\|K * f\|_{2}=\|m \widehat{f}\|_{2}$. We must be careful though. When, for example $m(\xi)=|\xi|^{i}$, then the corresponding SIO is not given by a principal value integral, though it can be obtained as a limit of a particular sequence of $\epsilon_{n} \rightarrow 0$. (See p. 51 of [86]).

There is an excellent account of convolution SIOs in the classic [86].

For square functions, Plancherel's theorem is also applicable in the convolution case when $Q_{t} f=\psi_{t} * f$ with $\psi_{t}(x)=t^{-n} \psi(x) / t$ where $\psi \in C_{0}^{\infty}\left(\mathbb{R}^{n}\right)$ with $\int_{\mathbb{R}^{n}} \psi(x) d x=0$ as discussed in (1.24). The key point is that the Fourier transform $\widehat{\psi}$ belongs to the test space $\mathcal{S}\left(\mathbb{R}^{n}\right)$ of rapidly decreasing $C^{\infty}$ functions and $\widehat{\psi}(0)=\int_{0}^{\infty} \psi(x) d x=0$, so that

$$
|\widehat{\psi}(\xi)| \lesssim \min \left\{|\xi|,|\xi|^{-1}\right\}
$$

for all $\xi \in \mathbb{R}^{n}$. Moreover $\widehat{\psi}_{t}(\xi)=\widehat{\psi}(t \xi)$. Then, using (1.22),

$$
\begin{aligned}
& \left\|S_{\psi} f\right\|_{2} \approx\left\|G_{\psi} f\right\|_{2}=\iint_{\mathbb{R}_{+}^{n+1}}\left|Q_{t} f(x)\right|^{2} \frac{d x d t}{t} \\
& =\int_{0}^{\infty} \int_{\mathbb{R}^{n}}|\widehat{\psi}(t \xi)|^{2}|\widehat{f}(\xi)|^{2} d \xi \frac{d t}{t}=\int_{\mathbb{R}^{n}}|\widehat{f}(\xi)|^{2}\left(\int_{0}^{\infty}|\widehat{\psi}(t \xi /|\xi|)|^{2} \frac{d t}{t}\right) d \xi \\
& \lesssim\|f\|_{L^{2}\left(\mathbb{R}^{n}\right)}^{2}\left(\int_{0}^{1} t^{2} \frac{d t}{t}+\int_{1}^{\infty} t^{-2} \frac{d t}{t}\right) \lesssim\|f\|_{2} .
\end{aligned}
$$


We remark for later use that if $\psi$ is a non-trivial real-valued radial function, then $\widehat{\psi}$ has the same properties, so with a slight abuse of notation we have

$$
\int_{0}^{\infty}|\widehat{\psi}(t \xi)|^{2} \frac{d t}{t}=\int_{0}^{\infty}|\widehat{\psi}(t|\xi|)|^{2} \frac{d t}{t}=\int_{0}^{\infty}|\widehat{\psi}(t)|^{2} \frac{d t}{t}=c<\infty
$$

and by renormalizing, we may suppose that $c=1$. In this case $\left\|G_{\psi} f\right\|_{2}=\|f\|_{2}$.

On noting that $Q_{t}$ is self-adjoint in this case, we obtain that

$$
\int_{0}^{\infty} Q_{s}^{2} \frac{d s}{s}=\mathfrak{I}
$$

in the strong operator topology on $L^{2}$, where $\mathfrak{I}$ is the identity operator on $L^{2}\left(\mathbb{R}^{n}\right)$. The identity (3.2) is referred to as the Calderón reproducing formula.

\subsection{The non-convolution case}

The non-convolution case is much more subtle. The original proofs of the $L^{2}$ boundedness of the prototypical non-convolution examples (1.7) and (1.9), had each been a real tour de force, obtained by a variety of methods. See [25] for the first Calderón commutator, [35] for the second commutator, [36] for the higher commutators, [27] for the Cauchy integral on a Lipschitz graph with small Lipschitz constant, and [39] for the Cauchy integral on all Lipschitz graphs. Thus, the search for general $L^{2}$ boundedness criteria was driven in large part by the desire to better understand these fundamentally important examples and to treat them in a more systematic way.

The first such criterion was the $T 1$ Theorem of David and Journé [44]:

Theorem 3.3 (T1 Theorem) [44]. Suppose that $T$ is a singular integral operator associated to a standard kernel $K(x, y)$ satisfying (1.2), (1.3). Then $T$ extends to a bounded operator on $L^{2}$ if and only if $T$ satisfies $W B P$ (1.13), and $T 1 \in B M O$ and ${ }^{t} T 1 \in B M O$.

Remark If $K(x, y)$ is antisymmetric, and $T$ is the associated principal value operator given by (1.12), then ${ }^{t} T=-T$, and WBP is satisfied automatically, as we have observed above (cf. (1.12), (1.13)). Thus, in that case, matters reduce to the simple statement that $T: L^{2} \rightarrow L^{2} \Longleftrightarrow T 1 \in B M O$.

Remark Note that the "only if" direction of the $T 1$ Theorem was already known: given that $T$ is $L^{2}$ bounded, one obtains that $T 1,{ }^{t} T 1 \in B M O$ by the Peetre-Spanne-Stein Theorem, and as we have noted earlier, WBP follows by Cauchy-Schwarz.

Remark The $T 1$ Theorem yields a simple proof of the $L^{2}$ boundedness of the first Calderón commutator $C_{A}^{1}$, for $A$ a Lipschitz function. Here is a formal proof, which can easily be made rigorous. By antisymmetry, it is enough to verify that $C_{A}^{1} 1 \in B M O$. By the representation (1.8), and the fact that $(d / d x \circ H) 1=0$, we have that $C_{A}^{1} 1=\frac{1}{i} H A^{\prime}$. By the Peetre-Spanne-Stein Theorem, the latter belongs to $B M O$, since $A^{\prime} \in L^{\infty}$ and $H: L^{2} \rightarrow L^{2}$. Similarly, one may handle the higher order commutators by induction, reducing $C_{A}^{k} 1$ to $C_{A}^{k-1} A^{\prime}$. 
We now present the proof of the $T 1$ Theorem, following an approach made explicit in [33], although many of the essential ideas were already implicit in [38]. We begin with an analogue of Theorem 3.3 for square functions, which appears in [33].

Theorem 3.4 (T1 Theorem for square functions) [33]. Let

$$
\Theta_{t} f(x):=\int_{\mathbb{R}^{n}} \psi_{t}(x, y) f(y) d y,
$$

where $\psi_{t}(x, y)$ satisfies (1.14), (1.15). Suppose that $d \mu(x, t):=\left|\Theta_{t} 1(x)\right|^{2} d x d t / t$ is a Carleson measure, i.e., that

$$
\sup _{Q} \frac{1}{|Q|} \int_{0}^{\ell(Q)} \int_{Q}\left|\Theta_{t} 1(x)\right|^{2} \frac{d x d t}{t}=:\|\mu\|_{\mathcal{C}}<\infty .
$$

Then the following square function estimate holds:

$$
\left\|G_{\psi} f\right\|_{L^{2}\left(\mathbb{R}^{n}\right)}^{2}=\iint_{\mathbb{R}_{+}^{n+1}}\left|\Theta_{t} f(x)\right|^{2} \frac{d x d t}{t} \lesssim\|f\|_{L^{2}\left(\mathbb{R}^{n}\right)}^{2} .
$$

Remark The converse direction (i.e. that (3.6) implies (3.5)) is essentially due to Fefferman and Stein [51].

Proof of Theorem 3.4 We start with the special case when $\Theta_{t} 1 \equiv 0$ for all $t>0$. We shall show that, in this case, $\Theta_{t}$ satisfies the orthogonality condition

$$
\left\|\Theta_{t} Q_{s}\right\|_{L^{2} \rightarrow L^{2}} \lesssim \min \left(\frac{s}{t}, \frac{t}{s}\right)^{\eta} \quad \text { for some } \eta>0 \text {, }
$$

with respect to convolution operators $Q_{s}$ defined by $Q_{s} f:=\zeta_{s} * f$ with $\zeta_{s}(x):=$ $s^{-n} \zeta(x / s)$ with $\zeta \in C_{0}^{\infty}(B(0,1))$ and $\int \zeta=0$. To do this, we shall apply the Schur estimate (1.32) to the kernel $K_{t s}(x, y)=\int \psi_{t}(x, z) \zeta_{s}(z-y) d z$ of $\Theta_{t} Q_{s}$.

When $s \leq t$, we use the smoothness (1.15) of $\psi_{t}(x, y)$ and cancellation of $\zeta_{s}$ to estimate

$$
\begin{aligned}
\int_{\mathbb{R}^{n}}\left|K_{t s}(x, y)\right| d y & =\int_{\mathbb{R}^{n}}\left|\int_{|z-y| \leq s}\left(\psi_{t}(x, z)-\psi_{t}(x, y)\right) \zeta_{s}(z-y) d z\right| d y \\
& \lesssim \int_{\mathbb{R}^{n}} \sup _{\{z ;|z-y| \leq s\}}\left|\psi_{t}(x, z)-\psi_{t}(x, y)\right| d y \\
& \lesssim \int_{\mathbb{R}^{n}} \frac{s^{\alpha}}{(t+|x-y|)^{n+\alpha}} d y \lesssim \frac{s^{\alpha}}{t^{\alpha}}
\end{aligned}
$$

for all $x \in \mathbb{R}^{n}$. Also, using (1.19),

$$
\int_{\mathbb{R}^{n}}\left|K_{t s}(x, y)\right| d x \leq \int_{\mathbb{R}^{n}} \int_{\mathbb{R}^{n}}\left|\psi_{t}(x, z)\right|\left|\zeta_{s}(z-y)\right| d x d z \lesssim 1
$$


for all $y \in \mathbb{R}^{n}$, so the Schur estimate (1.32) gives $\left\|\Theta_{t} Q_{s}\right\|_{o p} \lesssim(s / t)^{\alpha / 2}$.

When $t \leq s$, we reverse the roles of smoothness and cancellation, so in fact here is where we use $\Theta_{t} 1 \equiv 0$. For all $y \in \mathbb{R}^{n}$ :

$$
\begin{aligned}
\int_{\mathbb{R}^{n}}\left|K_{t s}(x, y)\right| d x= & \int_{\mathbb{R}^{n}}\left|\int_{\mathbb{R}^{n}} \psi_{t}(x, z)\left(\zeta_{s}(z-y)-\zeta_{s}(x-y)\right) d z\right| d x \\
\leq & \int_{\mathbb{R}^{n}} \int_{|x-z|>s}\left|\psi_{t}(x, z) \zeta_{s}(z-y)\right| d z d x \\
& +\int_{\mathbb{R}^{n}} \int_{|x-z|>s}\left|\psi_{t}(x, z) \zeta_{s}(x-y)\right| d z d x \\
& +\int_{\mathbb{R}^{n}} \int_{|x-z| \leq s}\left|\psi_{t}(x, z)\right|\left|\zeta_{s}(z-y)-\zeta_{s}(x-y)\right| d z d x,
\end{aligned}
$$

so that in turn,

$$
\begin{aligned}
\int_{\mathbb{R}^{n}}\left|K_{t s}(x, y)\right| d x \lesssim & \int_{\mathbb{R}^{n}} \frac{s}{(s+|z-y|)^{n+1}}\left(\int_{|x-z|>s} \frac{t^{\alpha}}{|x-z|^{n+\alpha}} d x\right) d z \\
& +\int_{\mathbb{R}^{n}} \frac{s}{(s+|x-y|)^{n+1}}\left(\int_{|x-z|>s} \frac{t^{\alpha}}{|x-z|^{n+\alpha}} d z\right) d x \\
& +\frac{t^{\alpha / 2}}{s^{\alpha / 2}} \int_{\mathbb{R}^{n}} \frac{s^{\alpha / 2}}{(s+|x-y|)^{n+\alpha / 2}}\left(\int_{\mathbb{R}^{n}} \frac{t^{\alpha / 2}|x-z|^{\alpha / 2}}{(t+|x-z|)^{n+\alpha}} d z\right) d x \lesssim \frac{t^{\alpha / 2}}{s^{\alpha / 2}} .
\end{aligned}
$$

(We have used the fact, noted after (1.24), that the family $\left\{\zeta_{s}(x-y)\right\}$ satisfies the Littlewood Paley bounds (1.14) and (1.15) for all $\alpha \leq 1$.)

Continuing as above we obtain $\left\|\Theta_{t} Q_{s}\right\|_{o p} \lesssim(t / s)^{\alpha / 4}$, thus completing the proof of the orthogonality condition (3.7).

We further recall that $\left\|G_{\zeta} f\right\|_{2}=\int_{0}^{\infty}\left\|Q_{s} f\right\|_{2}^{2} \lesssim\|f\|_{2}$, as we have shown in (3.1). On choosing $\zeta$ to be a non-trivial real-valued radial function, appropriately normalised, we have the Calderón reproducing formula

$$
\int_{0}^{\infty} Q_{s}^{2} \frac{d s}{s}=\mathfrak{I},
$$

as shown in (3.2).

We are now ready to dispose of the special case when $\Theta_{t} 1=0$. Indeed

$$
\begin{aligned}
\iint_{\mathbb{R}_{+}^{n+1}}\left|\Theta_{t} f(x)\right|^{2} \frac{d x d t}{t}= & \int_{0}^{\infty}\left\|\Theta_{t} f\right\|_{2}^{2} \frac{d t}{t} \\
= & \int_{0}^{\infty}\left\|\int_{0}^{\infty}\left(\Theta_{t} Q_{s}\right) Q_{s} f \frac{d s}{s}\right\|_{2}^{2} \frac{d t}{t} \\
\leq & \sup _{t>0} \int_{0}^{\infty}\left\|\Theta_{t} Q_{s}\right\|_{o p} \frac{d s}{s} \sup _{s>0} \int_{0}^{\infty}\left\|\Theta_{t} Q_{s}\right\|_{o p} \frac{d t}{t} \\
& \times \int_{0}^{\infty}\left\|Q_{s} f\right\|_{2}^{2} \frac{d s}{s} \\
& \lesssim\|f\|_{2}^{2}
\end{aligned}
$$


where we have used (3.2) in the second line, a variant of the Schur inequality (1.32) in the third line, and (3.7) and (3.1) in the final one.

We now return to the proof of Theorem 3.4 in the general case. To this end, we use a technique from [38], which has become standard in this subject. Let $P_{t}$ be a nice approximate identity, i.e., $P_{t} f:=\varphi_{t} * f$, where $\varphi_{t}(x):=t^{-n} \varphi(x / t)$, and $0 \leq \varphi \in C_{0}^{\infty}(B(0,1))$, with $\int \varphi=1$, so that $P_{t} 1=1$. Write

$$
\Theta_{t}=\left(\Theta_{t}-\left(\Theta_{t} 1\right) P_{t}\right)+\left(\Theta_{t} 1\right) P_{t}=: R_{t}+\left(\Theta_{t} 1\right) P_{t}
$$

Then $R_{t} 1 \equiv 0$ for all $t>0$, and, since $\Theta_{t} 1 \in L^{\infty}\left(\mathbb{R}^{n}\right)$ uniformly in $t>0$ by (1.17), the kernel of $R_{t}$ continues to satisfy (1.14), (1.15). The contribution of $R_{t}$ may therefore be handled by the special case that we have just proved.

The term $\left(\Theta_{t} 1\right) P_{t}$ may be treated by Carleson's embedding lemma [29]:

$$
\begin{aligned}
\iint_{\mathbb{R}_{+}^{n+1}}\left|\Theta_{t} 1(x)\right|^{2}\left|P_{t} f(x)\right|^{2} \frac{d x d t}{t} & =: \iint_{\mathbb{R}_{+}^{n+1}}\left|P_{t} f(x)\right|^{2} d \mu(x, t) \\
& \leq C\|\mu\|_{\mathcal{C}}\left\|N_{*}\left(P_{t} f\right)\right\|_{L^{2}\left(\mathbb{R}^{n}\right)}^{2}
\end{aligned}
$$

where $\mu$ and $\|\mu\|_{\mathcal{C}}$ are defined in the statement of the theorem and

$$
N_{*} F(x):=\sup _{\{(y, t):|x-y|<t\}}|F(y, t)|
$$

is the non-tangential maximal function. In turn, it is a routine matter to verify that $N_{*}\left(P_{t} f\right)(x) \lesssim M f(x)$ whence $\left\|N_{*}\left(P_{t} f\right)\right\|_{L^{2}\left(\mathbb{R}^{n}\right)} \lesssim\|M f\|_{L^{2}\left(\mathbb{R}^{n}\right)} \lesssim\|f\|_{L^{2}\left(\mathbb{R}^{n}\right)}$ by (1.30). The proof of Theorem 3.4 is now complete.

Remark 3.10 The lemma of Carleson which we have just applied, is of fundamental importance in analysis. There is a proof in Chapter 2, Section 2, of [87].

Remark 3.11 For later use, we note that our proof of the $T 1$ theorem for square functions, Theorem 3.4, remains valid when the Hölder condition (1.15) is replaced by the following weaker integral condition: There exists $\alpha>0, C<\infty$, such that for all $x \in \mathbb{R}^{n}$ and all $0<s \leq t<\infty$,

$$
\int_{\mathbb{R}^{n}\{z ;|z-y| \leq s\}} \sup _{t}\left|\psi_{t}(x, z)-\psi_{t}(x, y)\right| d y \leq C \frac{s^{\alpha}}{t^{\alpha}} .
$$

Proof of Theorem 3.3 With Theorem 3.4 in hand, we now turn to the proof of the $T 1$ theorem. As in the proof of Theorem 3.4, let $Q_{s}$ denote convolution operators of the form $Q_{s} f=\zeta_{s} * f$ with $\zeta_{s}(x):=s^{-n} \zeta(x / s)$ for $s>0$, where $\zeta$ is a real-valued radial function in $C_{0}^{\infty}(B(0,1))$ with $\int \zeta=0$, normalised so that (3.2) holds. The interested reader may readily verify that the operators

$$
P_{t}:=\int_{t}^{\infty} Q_{s}^{2} \frac{d s}{s}
$$

form a nice approximate identity in the sense that $P_{t} f=\varphi_{t} * f$ with $\varphi_{t}(x)=$ $t^{-n} \varphi(x / t)$, where $\varphi \in C_{0}^{\infty}(B(0,2))$ with $\int \varphi=1$. 
Now, for $T$ as in Theorem 3.3, f, $g \in C_{0}^{\infty}$, and $P_{t}$ as in (3.13), we may write

$$
\begin{aligned}
\langle T f, g\rangle & =\lim _{\varepsilon \rightarrow 0}\left\langle T P_{\varepsilon} f, P_{\varepsilon} g\right\rangle=-\lim _{\varepsilon \rightarrow 0} \int_{\varepsilon}^{1 / \varepsilon} \frac{d}{d t}\left\langle T P_{t} f, P_{t} g\right\rangle d t \\
& =\lim _{\varepsilon \rightarrow 0} \int_{\varepsilon}^{1 / \varepsilon}\left\langle T P_{t} f, Q_{t}^{2} g\right\rangle \frac{d t}{t}+\lim _{\varepsilon \rightarrow 0} \int_{\varepsilon}^{1 / \varepsilon}\left\langle T Q_{t}^{2} f, P_{t} g\right\rangle \frac{d t}{t}=: I+I I,
\end{aligned}
$$

where we have used the WBP (1.13) to obtain the the fact that, as $R \rightarrow \infty$,

$$
\begin{aligned}
\left|\left\langle T P_{R} f, P_{R} g\right\rangle\right| & \lesssim R^{n}\left(\left\|P_{R} f\right\|_{\infty}+R\left\|\nabla P_{R} f\right\|_{\infty}\right)\left(\left\|P_{R} g\right\|_{\infty}+R\left\|\nabla P_{R} g\right\|_{\infty}\right) \\
& \lesssim R^{-n}\|f\|_{1}\|g\|_{1} \rightarrow 0 .
\end{aligned}
$$

(For the $L^{\infty}$ bounds, use $\left\|P_{R} f\right\|_{\infty}=\left\|\phi_{R} * f\right\|_{\infty} \leq\left\|\phi_{R}\right\|_{\infty}\|f\|_{1} \lesssim R^{-N}\|f\|_{1}$, etc.)

The dual of term $I I$ is the same as term $I$, except with ${ }^{t} T$ in place of $T$ and the roles of $f$ and $g$ reversed, so it is enough to treat term $I$. Since $Q_{t}$, having a radial kernel, is its own transpose, we therefore have (applying (3.1)) that

$$
\begin{aligned}
|I| & \leq\left(\iint_{\mathbb{R}_{+}^{n+1}}\left|\Theta_{t} f(x)\right|^{2} \frac{d x d t}{t}\right)^{1 / 2}\left(\iint_{\mathbb{R}_{+}^{n+1}}\left|Q_{t} g(x)\right|^{2} \frac{d x d t}{t}\right)^{1 / 2} \\
& \lesssim\left(\iint_{\mathbb{R}_{+}^{n+1}}\left|\Theta_{t} f(x)\right|^{2} \frac{d x d t}{t}\right)^{1 / 2}\|g\|_{2}
\end{aligned}
$$

where $\Theta_{t}=Q_{t} T P_{t}$, i.e., $\Theta_{t} f(x):=\int_{\mathbb{R}^{n}} \psi_{t}(x, y) f(y) d y$, and

$$
\psi_{t}(x, y):=\left\langle\zeta_{t}(x-\cdot), T \varphi_{t}(\cdot-y)\right\rangle
$$

Here, as above, $\zeta_{t}$ and $\varphi_{t}$ are the kernels of $Q_{t}$ and $P_{t}$, respectively.

What remains is to bound the first factor by $\|f\|_{2}$, for we can then conclude that $T$ is a bounded operator in $L^{2}\left(\mathbb{R}^{n}\right)$. We do this by applying the $T 1$ Theorem for square functions, Theorem 3.4.

For this we must first show that $\psi_{t}(x, y)$ satisfies the standard kernel conditions (1.14) and (1.15). When $|x-y| \leq 8 t$ then (1.14) is a consequence of (1.13) as we now show:

$$
\begin{aligned}
\left|\psi_{t}(x, y)\right| & =\left|\left\langle\zeta_{t}(x-\cdot), T \varphi_{t}(\cdot-y)\right\rangle\right| \lesssim t^{n}\left(\left\|\zeta_{t}\right\|_{\infty}+t\left\|\nabla \zeta_{t}\right\|_{\infty}\right)\left(\left\|\varphi_{t}\right\|_{\infty}+t\left\|\nabla \varphi_{t}\right\|_{\infty}\right) \\
& \leq c t^{-n} \approx \frac{t^{\alpha}}{(t+|x-y|)^{n+\alpha}}
\end{aligned}
$$

while (1.15) can be verified in a similar way. When $|x-y|>8 t$, we may use the representation (1.1) along with (1.3) and the fact that $\int \zeta_{s}=0$ to argue as in (2.6) to prove the claim:

$$
\begin{aligned}
\left|\psi_{t}(x, y)\right| & =\left|\int_{\mathbb{R}^{n}} \int_{\mathbb{R}^{n}} \zeta_{t}(x-w)(K(w, z)-K(x, z)) \varphi_{t}(z-y) d w d z\right| \\
& \leq C \sup \left\{\frac{|w-x|^{\alpha}}{|w-z|^{n+\alpha}}:|x-w| \leq t,|z-y| \leq 2 t\right\}
\end{aligned}
$$




$$
\begin{aligned}
& \times \int_{\mathbb{R}^{n}}\left|\zeta_{t}(x-w)\right| d w \int_{\mathbb{R}^{n}}\left|\varphi_{t}(z-y)\right| d z \\
\lesssim & \frac{t^{\alpha}}{|x-y|^{n+\alpha}} \approx \frac{t^{\alpha}}{(t+|x-y|)^{n+\alpha}} \quad\left(\text { using }|w-z|>\frac{1}{2}|x-y|\right) ;
\end{aligned}
$$

while (1.15) can be verified in a similar way.

Finally, we need to establish the Carleson measure bound (3.5):

$$
\sup _{Q} \frac{1}{|Q|} \int_{0}^{\ell(Q)} \int_{Q}\left|\Theta_{t} 1(x)\right|^{2} \frac{d x d t}{t}=:\|\mu\|_{\mathcal{C}}<\infty
$$

In fact, since $P_{t} 1=1$, we have

$$
\Theta_{t} 1=Q_{t}(T 1)=Q_{t} b
$$

where $b:=T 1 \in B M O$ by hypothesis. It is enough to invoke a simple version of the Fefferman-Stein inequality [51]:

$$
\begin{aligned}
\frac{1}{|Q|} \int_{0}^{\ell(Q)} & \int_{Q}\left|Q_{t} b(x)\right|^{2} \frac{d x d t}{t} \\
& =\frac{1}{|Q|} \int_{0}^{\ell(Q)} \int_{Q}\left|Q_{t}\left(\left(b-[b]_{3 Q}\right) 1_{3 Q}\right)(x)\right|^{2} \frac{d x d t}{t} \\
& \leq C \frac{1}{|Q|} \int_{3 Q}\left|b-[b]_{3 Q}\right|^{2} \leq C\|b\|_{*}^{2}
\end{aligned}
$$

where in the middle line we have used that $Q_{t} 1=0$ and that $\zeta_{t}(x-\cdot)$ is supported in the ball $B(x, t)$ with $t \leq \ell(Q)$, and in the last line we have used the $L^{2}$ bound (3.1) and the consequence (2.7) of the John-Nirenberg inequality.

This concludes the proof of the $T 1$ Theorem.

The $T 1$ theorem shed considerable light on the behavior of the Calderón commutators $C_{A}^{k}$ (1.7). Indeed, as we have remarked above, the $T 1$ theorem provided a general framework which incorporated the fundamental result of Calderón [25] concerning the $L^{2}$ boundedness of the first commutator $C_{A}^{1}$. Moreover, an induction scheme based on the $T 1$ theorem produces an operator norm of the order of $c^{k}$ (for some constant $c$ ) for the $\mathrm{k}^{\mathrm{th}}$ commutator $C_{A}^{k}$. Thus, expanding the operator $\mathfrak{C}_{A}$ in (1.9) as a series of commutators:

$$
\mathfrak{C}_{A}=\sum_{k=0}^{\infty}(-i)^{k} C_{A}^{k}
$$

one obtains a proof of the boundedness of the Cauchy integral on a Lipschitz curve with small Lipschitz constant, first proved by Calderón in [27]. However, the $T 1$ theorem does not yield a direct proof of the $L^{2}$ boundedness of the Cauchy integral operator on an arbitrary Lipschitz curve, which was first established by Coifman, McIntosh 
and Meyer, by showing that the norms of the commutators $C_{A}^{k}$ actually depend polynomially on $k$. [39]. The desire to rectify this shortcoming of the $T 1$ theorem, and to develop a general theory that would encompass the Cauchy integral operator, led to the so-called $T b$ Theorem.

The $T b$ theorem is an extension of the $T 1$ theorem, in which the conditions $T 1,{ }^{t} T 1 \in B M O$ are replaced by the conditions $T b_{1},{ }^{t} T b_{2} \in B M O$ for suitable functions $b_{1}, b_{2} \in L^{\infty}\left(\mathbb{R}^{n}\right)$. It was first proved in a special case by McIntosh and Meyer [75], and in general by David, Journé and Semmes [45].

Theorem 3.14 (Tb Theorem) [45]. Suppose that $b_{1}, b_{2} \in L^{\infty}$ are accretive, i.e., there is a constant $\delta>0$ such that

$$
\Re e\left(b_{i}\right)>\delta, \quad i=1,2 .
$$

Let $T$ be a mapping from $b_{1} C_{0}^{\infty}$ into $\left(b_{2} C_{0}^{\infty}\right)^{\prime}$, associated to a standard kernel $K(x, y)$ (equivalently, $b_{2} T b_{1}$ is a mapping from test functions to distributions associated to the kernel $b_{2}(x) K(x, y) b_{1}(y)$.) Suppose also that $b_{2} T b_{1}$ satisfies WBP (1.13) and that $T b_{1}$ and ${ }^{t} T b_{2}$ are in BMO. Then $T$ extends to a bounded operator on $L^{2}\left(\mathbb{R}^{n}\right)$.

Remark For the principal value operator associated to an antisymmetric kernel, it is enough to verify that there is a single accretive $b$ such that $T b \in B M O$. In this case $W B P$ holds automatically for $b T b$.

Remark The accretivity condition (3.15) may be relaxed to pseudo-accretivity:

$$
\inf _{Q}\left|[b]_{Q}\right| \geq \delta
$$

or even para-accretivity, a relaxed version of pseudo-accretivity in which nondegeneracy of the average over each given cube is replaced by nondegeneracy of the average over some sub-cube of comparable size.

Remark Somewhat earlier, it was proved in [75] that when $T b_{1}$ and ${ }^{t} T b_{2}$ both satisfy WBP, and $T b_{1}=0={ }^{t} T b_{2}$, then $T$ extends to a bounded operator on $L^{2}\left(\mathbb{R}^{n}\right)$. (In fact, this paper only mentions the case $b_{1}=b_{2}$, though the same proof holds when $b_{1}$ and $b_{2}$ are different functions [73].)

Both papers [75] and [45] provide alternative proofs of the Cauchy integral theorem of [39]. Indeed, let $\gamma$ be the graph of a Lipschitz function $A$, and observe that $b:=1+i A^{\prime}$ is accretive. By definition, (cf. (1.9), (1.10)),

$$
\mathfrak{C}_{A} b(x)=\left(\mathcal{C}_{\gamma} 1\right)(x+i A(x))
$$

and at least formally, by the formula of Plemelj, $\mathfrak{C}_{A} b=0$ in the sense of BMO. Moreover, the requisite operators satisfy WBP. (Some care must be taken in interpreting the Plemelj formula on an infinite graph, but this can be managed.)

The $T b$ theorem also provided a more direct proof of $n$ dimensional analogues of the Cauchy integral theorem, which had initially been proved by using the Calderón rotation method to extend the one dimensional theory to higher dimensions. In particular, 
such results include $L^{2}$ bounds for double layer potential operators, and derivatives of single layer potential operators, on strongly Lipschitz surfaces, thus allowing the methods of potential theory to be used to solve boundary value problems for harmonic functions on domains with such boundaries. There is a vast literature on singular integrals and potential theory, as developed for example by the school of Mikhlin [76] and many others. Calderón's paper [27] provided $L^{2}$ bounds for potential operators on surfaces with small Lipschitz constants, and was used by Fabes, Jodeit and Riviére to [50] to solve Dirichlet and Neumann problems on $C^{1}$ domains. After the publication of [39], Verchota [89] showed how to solve these boundary value problems for harmonic functions on all regions in $\mathbb{R}^{n}$ with strongly Lipschitz boundaries, using appropriate Rellich identities to invert the boundary potential operators, thus complementing the approach, via harmonic measures, of Dahlberg, Jerison, Kenig and others.

We shall not present the proof of the $T b$ theorem in the present survey, but here is the basic idea: one constructs a discrete variant of the Calderón reproducing formula (3.2) that is adapted to the accretive functions $b_{1}$ and $b_{2}$, and for which discrete square function estimates still hold for the adapted $Q_{t}$ operators. In the discrete version, they are now $Q_{k}$ 's. The main outline of the proof then follows that of the $T 1$ Theorem. We refer the reader to [34] or to [32], pp 64-67, for the details of an argument that is somewhat simpler than the original one in [45].

We shall however, present the proof of a square function version of the $T b$ theorem, due to Semmes [82], as it contains the germ of an idea that has turned out to be quite useful and to which we will return in the next section. The theorem generalizes Theorem 3.4, and the latter will be used in the course of the proof.

Theorem 3.16 ( $\mathrm{Tb}$ Theorem for square functions) [82] Let

$$
\Theta_{t} f(x):=\int \psi_{t}(x, y) f(y) d y,
$$

and assume that $\psi_{t}$ satisfies the standard kernel conditions (1.14), (1.15). Suppose there exists an accretive function $b$ such that $d \mu(x, t):=\left|\Theta_{t} b(x)\right|^{2} d x d t / t$ is a Carleson measure, i.e., that

$$
\sup _{Q} \frac{1}{|Q|} \int_{0}^{\ell(Q)} \int_{Q}\left|\Theta_{t} b(x)\right|^{2} \frac{d x d t}{t}=:\|\mu\|_{\mathcal{C}}<\infty .
$$

Then the square function estimate (3.6) holds:

$$
\left\|G_{\psi} f\right\|_{L^{2}\left(\mathbb{R}^{n}\right)}^{2}=\iint_{\mathbb{R}_{+}^{n+1}}\left|\Theta_{t} f(x)\right|^{2} \frac{d x d t}{t} \lesssim\|f\|_{L^{2}\left(\mathbb{R}^{n}\right)}^{2} .
$$

Proof By Theorem 3.4, it is enough to show that $\left|\Theta_{t} 1(x)\right|^{2} \frac{d x d t}{t}$ is a Carleson measure. By accretivity, we have that

$$
\left|\Theta_{t} 1\right| \leq C\left|\left(\Theta_{t} 1\right) P_{t} b\right|
$$


where $P_{t}$ is a nice approximate identity (e.g., as in (3.8)), with kernel supported in a ball of radius $t$. For each cube $Q$, let $b_{Q}:=b 1_{2} Q$, so that when $x \in Q$ and $t \leq \ell(Q)$, then $P_{t} b(x)=P_{t} b_{Q}(x)$. Therefore

$$
\begin{aligned}
& \frac{1}{|Q|} \int_{0}^{\ell(Q)} \int_{Q}\left|\Theta_{t} 1\right|^{2} \frac{d x d t}{t} \leq \frac{1}{|Q|} \int_{0}^{\ell(Q)} \int_{Q}\left|\left(\Theta_{t} 1\right) P_{t} b_{Q}\right|^{2} \frac{d x d t}{t} \\
& \quad \lesssim \frac{1}{|Q|} \int_{0}^{\ell(Q)} \int_{Q}\left|\Theta_{t} b_{Q}\right|^{2} \frac{d x d t}{t}+\frac{1}{|Q|} \int_{0}^{\ell(Q)} \int_{Q}\left|R_{t} b_{Q}\right|^{2} \frac{d x d t}{t}=I+I I
\end{aligned}
$$

where, again following [38] as in (3.8), we let $R_{t}=\Theta_{t}-\left(\Theta_{t} 1\right) P_{t}$. Now the kernels of $R_{t}$ satisfy (1.14), (1.15), and $R_{t}(1)=0$, so by the $T 1$ theorem for square functions,

$$
I I \leq \frac{1}{|Q|} \iint_{\mathbb{R}_{+}^{n+1}}\left|R_{t} b_{Q}\right|^{2} \frac{d x d t}{t} \lesssim \frac{1}{|Q|}\left\|b_{Q}\right\|_{2}^{2} \lesssim 1
$$

To bound the first term $I$, apply the assumption (3.17) together with the estimate

$$
\begin{aligned}
\int_{0}^{\ell(Q)} \int_{Q}\left|\Theta_{t}\left(b-b_{Q}\right)\right|^{2} \frac{d x d t}{t} & =\int_{0}^{\ell(Q)} \int_{Q}\left|\int_{\mathbb{R}^{n} \backslash 2 Q} \psi_{t}(x-y) b(y) d y\right|^{2} \frac{d x d t}{t} \\
& \lesssim \int_{0}^{\ell(Q)} \int_{Q}\left|\int_{\mathbb{R}^{n} \backslash 2 Q} \frac{t^{\alpha}}{|x-y|^{n+\alpha}} d y\right|^{2} \frac{d x d t}{t}\|b\|_{\infty}^{2} \lesssim|Q|
\end{aligned}
$$

Thus $\left|\Theta_{t} 1(x)\right|^{2} \frac{d x d t}{t}$ is a Carleson measure, and the proof is complete.

Remark 3.18 Observe that this argument carries over if $b$ is allowed to vary with $Q$, i.e. if we have a system $\left\{b_{Q}\right\}$, indexed on the dyadic cubes, satisfying

$$
\sup _{Q} \frac{1}{|Q|} \int_{0}^{\ell(Q)} \int_{Q}\left|\Theta_{t} 1\right|^{2} \frac{d x d t}{t} \leq C \sup _{Q} \frac{1}{|Q|} \int_{0}^{\ell(Q)} \int_{Q}\left|\left(\Theta_{t} 1\right) P_{t} b_{Q}\right|^{2} \frac{d x d t}{t}
$$

and

$$
\sup _{Q} \frac{1}{|Q|} \int_{0}^{\ell(Q)} \int_{Q}\left|\Theta_{t} b_{Q}\right|^{2} \frac{d x d t}{t} \leq C
$$

This observation is essentially due to Auscher and Tchamitchian [18], and is the starting point for the solution of the Kato problem, which we shall discuss in the next section.

Thus, it is natural to pose the question: when does (3.19) hold? In fact, the solution to the Kato problem provided a sufficient condition which answers this question (see in particular Theorem 4.7 (i), (iii), and Theorem 4.15 (i), (iii) below). Moreover, the question is related to some previous work of Christ [31], who gave the first example 
of a class of results which have come to be referred to as local $T b$ theorems. Here is a brief overview of the latter notion.

In some applications, it may not be at all evident that there is a single accretive (or pseudo-accretive) $b$ for which $T b$ is well behaved (where we should think of $T$ as either an SIO or a square function operator.) On the other hand, in such cases it is sometimes possible to find a family $\left\{b_{Q}\right\}$, indexed by dyadic cubes $Q$, such that $T b_{Q}$ behaves well locally on $Q$ as e.g. in (3.20). This motivates the introduction of the notion of a Local Tb Theorem, in which good local control of $T$, on each member of a family of suitably non-degenerate functions $b_{Q}$, one for each dyadic cube $Q$, still suffices to deduce global $L^{2}$ boundedness of $T$. Such results are the topic of the next section.

\section{Local $T b$ theorems and applications}

The first local $T b$ theorem was proved by Christ [31], in connection with the theory of analytic capacity. The appropriate version of non-degeneracy in this context is as follows: a pseudo-accretive system is a collection of functions $\left\{b_{Q}\right\}$, indexed by the dyadic cubes, with $b_{Q}$ supported in $Q$ and integrable, such that for some $\delta>0$, we have that

$$
\left|\int_{Q} b_{Q}\right| \geq \delta|Q| \text {. }
$$

Theorem 4.1 [31] Suppose that $T$ is a singular integral operator associated to a standard kernel $K(x, y)$, which in addition we assume to be in $L^{\infty}$. Suppose also that there are constants $\delta>0$ and $C_{0}<\infty$, and pseudo-accretive systems $\left\{b_{Q}^{1}\right\},\left\{b_{Q}^{2}\right\}$, with supp $b_{Q}^{i} \subseteq Q, i=1,2$, such that for each dyadic cube $Q$,

(i) $\left\|b_{Q}^{1}\right\|_{L^{\infty}(Q)}+\left\|b_{Q}^{2}\right\|_{L^{\infty}(Q)} \leq C_{0}$

(ii) $\left\|T b_{Q}^{1}\right\|_{L^{\infty}(Q)}+\left\|{ }^{t} T b_{Q}^{2}\right\|_{L^{\infty}(Q)} \leq C_{0}$

(iii) $\min \left\{\left|\int_{Q} b_{Q}^{1}\right|,\left|\int_{Q} b_{Q}^{2}\right|\right\} \geq \delta|Q|$.

Then $T$ extends to a bounded operator on $L^{2}$, with bound depending on $n, \delta, C_{0}$ and the kernel constants in (1.2), (1.3), but not on the $L^{\infty}$ norm of $K(x, y)$.

A few remarks are in order. The assumption that $K \in L^{\infty}$ is merely qualitative, and is satisfied, e.g., by smooth truncations of a standard kernel. This assumption allows one to make certain formal manipulations with impunity, during the course of the proof. Christ actually proved this theorem in the setting of a space $\mathcal{X}$ endowed with a pseudo-metric $\rho$ and a doubling measure $\mu$ (meaning that $\mu(B(x, 2 r)) \leq C \mu(x, r)$ for all $x \in \mathcal{X}$ and $r>0$ and some constant $C$ ), which, as he demonstrated, necessarily possesses a suitable version of a dyadic cube structure. Christ's theorem and the technique of its proof are related to the solution of Painlevé's problem concerning the characterization of those compact sets $K \subset \mathbb{C}$ for which there exist non-constant bounded analytic functions on $\mathbb{C} \backslash K$. We will not discuss the latter subject in detail, nor the deep related work on extending $T b$ theory to the non-doubling setting. See Section 5 for some further results in this vein. 
Instead, we shall concentrate on extensions of Christ's result in another direction, in which $L^{\infty}$ control of $b_{Q}$ and $T b_{Q}$ is replaced by local, scale invariant $L^{2}$ control. Moreover, our emphasis will be on local $T b$ theory for square functions, as opposed to singular integrals, although in Section 5 we shall discuss briefly some recent progress in the latter case. It turns out that the square function setting is somewhat technically simpler, yet, to date, it is that setting which has been more fruitful in terms of applications.

Before presenting the local $T b$ theorem for square functions, we introduce the dyadic averaging operator $A_{t}$, defined by

$$
A_{t} f(x):=\frac{1}{|Q(x, t)|} \int_{Q(x, t)} f(y) d y,
$$

where $Q(x, t) \in \mathbb{D}$ denotes the minimal dyadic cube containing $x$, with side length at least $t$. Just as the nice approximate identity $P_{t}$ defined before (3.8) filters out frequencies higher than $1 / t$, so does $A_{t}$, the difference being that $A_{t} f$ approximates $f$ by a piecewise constant function, whereas $P_{t} f$ approximates $f$ by a smooth function. These approximations are close in the following sense:

\section{Lemma 4.3}

$$
\iint_{\mathbb{R}_{+}^{n+1}}\left|\left(A_{t}-P_{t}\right) f(x)\right|^{2} \frac{d x d t}{t} \lesssim\|f\|_{2}^{2}
$$

for all $f \in L^{2}\left(\mathbb{R}^{n}\right)$.

Proof Write the operator $A_{t}$ as an integral operator $A_{t} f(x)=\int_{\mathbb{R}^{n}} \chi_{t}(x, y) f(y) d y$ with

$$
\chi_{t}(x, y):=\frac{1}{|Q(x, t)|} 1_{Q(x, t)}(y),
$$

where $Q(x, t)$ is the unique dyadic cube containing $x$ with $\ell(Q) \geq t>\frac{1}{2} \ell(Q)$. It is easy to check that $\chi_{t}(x, y)$ satisfies the first Littlewood-Paley estimate (1.14), and although it does not satisfy (1.15), it does satisfy the integral version (3.12) presented in Remark 3.11 with $\alpha=1$. This is because, when $|z-y| \leq s \leq t$, then $\chi_{t}(x, z)-\chi_{t}(x, y)$ can only be non-zero when $\operatorname{dist}(y, \partial Q(x, t)) \leq s$. Here $\partial Q(x, t)$ denotes the boundary of $Q(x, t)$. Therefore

$$
\begin{aligned}
\int_{\mathbb{R}^{n}\{z ;|z-y| \leq s\}}\left|\chi_{t}(x, z)-\chi_{t}(x, y)\right| d y & \leq \int_{\operatorname{dist}(y, \partial Q(x, t)) \leq s} \frac{1}{|Q(x, t)|} d y \lesssim \frac{s t^{n-1}}{|Q(x, t)|} \\
& \approx \frac{s}{t}
\end{aligned}
$$

as required. The kernel $\varphi_{t}(x, y)=\varphi_{t}(x-y)$ of $P_{t}$ satisfies the standard LittlewoodPaley estimates (1.14) and (1.15), so the kernel $\left(\chi_{t}-\varphi_{t}\right)(x, y)$ of $\Theta_{t}:=A_{t}-P_{t}$ satisfies (1.14) and (3.12). Moreover $\Theta_{t} 1(x)=\int \chi_{t}(x, y) d y-1=0$ for all $x \in \mathbb{R}^{n}$, so by the special case when $\Theta_{t} 1 \equiv 0$ of the $T 1$ Theorem for square functions as generalised in Remark 3.11, the square function estimate (4.4) holds. 
Before coming to the local $T b$ theorem for square functions, we make two preliminary observations about Carleson measures. A Carleson measure is a Borel measure $\mu$ on $\mathbb{R}_{+}^{n+1}$ such that, for some constant $C_{\mu}<\infty, \mu\left(R_{Q}\right) \leq C_{\mu}|Q|$ for all cubes $Q \subset \mathbb{R}^{n}$, where $R_{Q}$ denotes the Carleson box $R_{Q}:=Q \times(0, \ell(Q)) \subset \mathbb{R}_{+}^{n+1}$.

In the first observation, we note that it is sufficient to check the Carleson measure condition on dyadic cubes:

Lemma 4.5 Let $\mu$ be a Borel measure on $\mathbb{R}_{+}^{n+1}$ such that $\mu\left(R_{Q}\right) \leq C|Q|$ for all $Q \in \mathbb{D}$, where $R_{Q}:=Q \times(0, \ell(Q))$. Then $\mu\left(R_{Q}\right) \leq 2^{2 n} C|Q|$ for all cubes $Q \subset \mathbb{R}^{n}$.

Proof For a cube $Q \subset \mathbb{R}^{n}$, cover $Q$ with $Q_{j} \in \mathbb{D}, j=1,2, \cdots, N$, with $N \leq 2^{n}$ and $\ell(Q) \leq \ell\left(Q_{j}\right)<2 \ell(Q)$. Then $R_{Q} \subset \bigcup_{j} R_{Q_{j}}$, so that

$$
\mu\left(R_{Q}\right) \leq \sum \mu\left(R_{Q_{j}}\right) \leq C \sum\left|Q_{j}\right| \leq C 2^{n} 2^{n}|Q|
$$

In the second, we show that when proving that a measure $\mu$ on $\mathbb{R}_{+}^{n+1}$ is a Carleson measure, it suffices to prove a bound for $\mu$ on an $\eta$-ample sawtooth region of each Carleson box $R_{Q}$ for some $\eta>0$. This is a region of the form $E_{Q}^{*}:=R_{Q} \backslash\left(\bigcup_{j} R_{Q_{j}}\right)$, where $\left\{Q_{j}\right\}$ is a collection of non-overlapping dyadic sub-cubes of $Q$ such that $E_{Q}:=$ $Q \backslash\left(\bigcup_{j} Q_{j}\right)$ has Lebesgue measure $\left|E_{Q}\right| \geq \eta|Q|$. This result is known as a JohnNirenberg type lemma for Carleson measures.

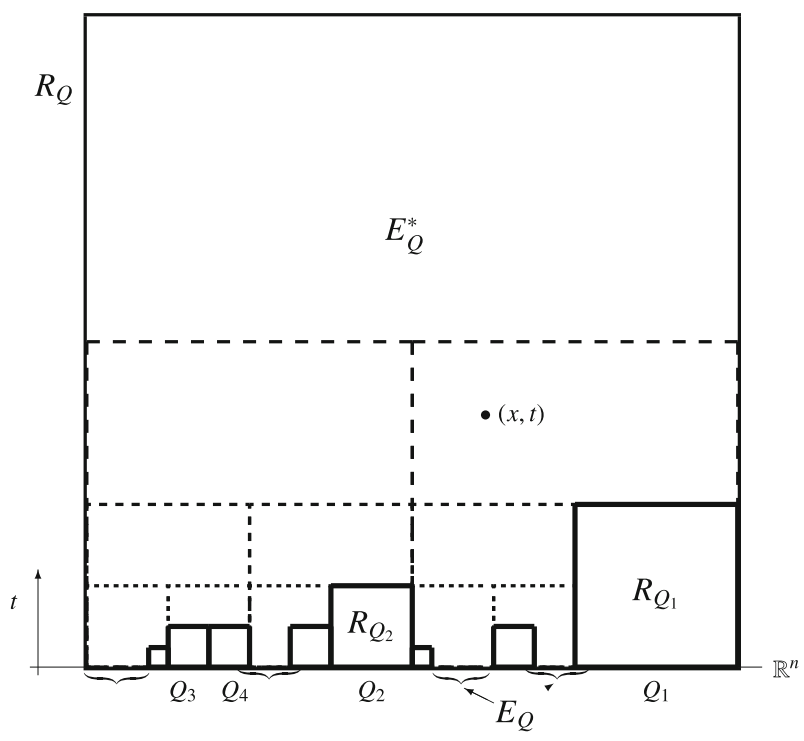


Lemma 4.6 ("John-Nirenberg" lemma for Carleson measures). Let $\mu$ be a Borel measure on $\mathbb{R}_{+}^{n+1}$. Suppose that there exist $\eta>0, C_{1}<\infty$ such that for every dyadic cube $Q$, there is a collection $\left\{Q_{j}\right\}$ of non-overlapping dyadic sub-cubes of $Q$ satisfying

$$
\left|E_{Q}\right|:=\left|Q \backslash\left(\cup_{j} Q_{j}\right)\right| \geq \eta|Q|
$$

for which the $\eta$-ample sawtooth $E_{Q}^{*}:=R_{Q} \backslash\left(\bigcup_{j} R_{Q_{j}}\right)$ satisfies

$$
\mu\left(E_{Q}^{*}\right) \leq C_{1}|Q|
$$

Then $\mu$ is a Carleson measure, with

$$
\sup _{Q \in \mathbb{D}} \frac{\mu\left(R_{Q}\right)}{|Q|} \leq \frac{C_{1}}{\eta} .
$$

Sketch of proof Start with a dyadic cube $Q$. Then $R_{Q}=E_{Q}^{*} \cup \cup_{j} R_{Q_{j}}$ (disjoint union), and $\sum_{j}\left|Q_{j}\right|=\left|\bigcup_{j} Q_{j}\right| \leq(1-\eta)|Q|$. Iterating, we decompose $R_{Q_{j}}=$ $E_{Q_{j}}^{*} \cup \bigcup_{k} R_{Q_{j, k}}$ (disjoint union) and

$$
\sum_{j, k}\left|Q_{j, k}\right| \leq \sum_{j}(1-\eta)\left|Q_{j}\right| \leq(1-\eta)^{2}|Q|
$$

and so on. Therefore

$$
\begin{aligned}
\mu\left(R_{Q}\right) & =\mu\left(E_{Q}^{*}\right)+\sum_{j} \mu\left(R_{Q_{j}}\right) \\
& \leq C_{1}|Q|+\sum_{j} \mu\left(E_{Q_{j}}^{*}\right)+\sum_{j, k} \mu\left(R_{Q_{j, k}}\right) \\
& \leq C_{1}|Q|+C_{1} \sum_{j}\left|Q_{j}\right|+\sum_{j, k} \mu\left(E_{Q_{j, k}}^{*}\right)+\cdots \\
& \leq C_{1}\left(|Q|+(1-\eta)|Q|+(1-\eta)^{2}|Q|+\cdots\right)=\frac{C_{1}|Q|}{\eta}
\end{aligned}
$$

\subsection{Local $T b$ theorems for square functions}

We begin with a local $T b$ theorem for square functions, which extends the global version, Theorem 3.16. This result is essentially contained in the solution of the Kato problem: see [60,56,9]. The stated version is formulated explicitly in [2] and [54].

Theorem 4.7 Let $\Theta_{t} f(x):=\int \psi_{t}(x, y) f(y) d y$, where $\psi_{t}(x, y)$ satisfies (1.14), (1.15). Suppose that there exist constants $\delta>0, C_{0}<\infty$, and a system $\left\{b_{Q}\right\}$ of functions indexed by dyadic cubes $Q$ in $\mathbb{R}^{n}$ such that for each dyadic cube $Q$ : 
(i) $\int_{\mathbb{R}^{n}}\left|b_{Q}(x)\right|^{2} d x \leq C_{0}|Q|$;

(ii) $\int_{0}^{\ell(Q)} \int_{Q}\left|\Theta_{t} b_{Q}(x)\right|^{2} \frac{d x d t}{t} \leq C_{0}|Q|$;

(iii) $\left|\int_{Q} b_{Q}(x) d x\right| \geq \delta|Q|$.

Then the following square function estimate holds:

$$
\iint_{\mathbb{R}_{+}^{n+1}}\left|\Theta_{t} f(x)\right|^{2} \frac{d x d t}{t} \lesssim\|f\|_{2}^{2}
$$

Remark 4.9 On dividing each function $b_{Q}$ by an appropriate complex constant, we may replace (iii) by

$$
\text { (iii') } \int_{Q} b_{Q}(x) d x=|Q|
$$

noting that the constant $C_{0}$ in (i), (ii) needs to be modified. We shall assume (i), (ii) and (iii') in the proof.

Proof We follow the outline of the proof of Semmes' result Theorem 3.16 (cf. [18] and Remark 3.18), but with an additional stopping time argument, in the spirit of that used in Christ's proof [31] of Theorem 4.1, to exploit the pseudo-accretive system condition, which is weaker than global pseudo-accretivity of a single function $b$.

As in the proof of Theorem 3.16, again by Theorem 3.4, it suffices to verify that $\left|\Theta_{t} 1\right|^{2} d x d t / t$ is a Carleson measure, now given the existence of a family $\left\{b_{Q}\right\}$ satisfying hypotheses (i), (ii) and (iii'). To this end, we first observe that, as in [82] and [18], it is enough to verify the bound

$$
\sup _{Q \in \mathbb{D}} \frac{1}{|Q|} \iint_{R_{Q}}\left|\Theta_{t} 1\right|^{2} \frac{d x d t}{t} \leq C_{2} \sup _{Q \in \mathbb{D}} \frac{1}{|Q|} \iint_{R_{Q}}\left|\left(\Theta_{t} 1\right) A_{t} b_{Q}\right|^{2} \frac{d x d t}{t},
$$

where $R_{Q}:=Q \times(0, \ell(Q))$ is the Carleson box above $Q$, and $A_{t}$ is the dyadic averaging operator defined in (4.2). Indeed, suppose momentarily that (4.10) holds. Then to obtain (3.5), and thus also the conclusion of the theorem, it suffices to show that the right hand side of (4.10) is bounded. Once again let $P_{t}$ define a nice approximate identity as defined before (3.8), and following [38], write

$$
\left(\Theta_{t} 1\right) P_{t} b_{Q}=\left(\left(\Theta_{t} 1\right) P_{t} b_{Q}-\Theta_{t} b_{Q}\right)+\Theta_{t} b_{Q}=: R_{t} b_{Q}+\Theta_{t} b_{Q}
$$

where $R_{t}:=\left(\Theta_{t} 1\right) P_{t}-\Theta_{t}$ satisfies $R_{t} 1 \equiv 0$. Therefore

$$
\begin{aligned}
\iint_{R_{Q}}\left|\left(\Theta_{t} 1\right) A_{t} b_{Q}(x)\right|^{2} \frac{d x d t}{t} \leq & \iint_{\mathbb{R}_{+}^{n+1}}\left|\left(\Theta_{t} 1\right)\left(A_{t}-P_{t}\right) b_{Q}(x)\right|^{2} \frac{d x d t}{t} \\
& +\iint_{\mathbb{R}_{+}^{n+1}}\left|R_{t} b_{Q}(x)\right|^{2} \frac{d x d t}{t}+\iint_{R_{Q}}\left|\Theta_{t} b_{Q}(x)\right|^{2} \frac{d x d t}{t} \\
& \lesssim \int_{\mathbb{R}^{n}}\left|b_{Q}(x)\right|^{2} d x+\int_{\mathbb{R}^{n}}\left|b_{Q}(x)\right|^{2} d x+|Q| \lesssim|Q|
\end{aligned}
$$

where the bound on the first of the three integrals follows from Lemma 4.3 and (1.17), the bound on the second follows from the special case of Theorem 3.4 since $R_{t} 1 \equiv 0$, and the bound on the third is hypothesis (ii). The final estimate needs (i). 
We turn now to the proof of (4.10). In order to apply Lemma 4.6, it suffices to show that there are constants $\eta>0, C<\infty$, such that for each $Q \in \mathbb{D}$, there is a dyadic sawtooth region

$$
E_{Q}^{*}:=R_{Q} \backslash\left(\cup_{j} R_{Q_{j}}\right),
$$

where $\left\{Q_{j}\right\}$ are non-overlapping dyadic sub-cubes of $Q$, with

$$
\left|Q \backslash\left(\cup_{j} Q_{j}\right)\right| \geq \eta|Q|
$$

and

$$
\iint_{E_{Q}^{*}}\left|\Theta_{t} 1(x)\right|^{2} \frac{d x d t}{t} \leq 4 \iint_{E_{Q}^{*}}\left|\left(\Theta_{t} 1(x)\right)\left(A_{t} b_{Q}(x)\right)\right|^{2} \frac{d x d t}{t} .
$$

We prove (4.12) via the same stopping time argument as in $[60,56,9]$. See also [31], where a similar idea had previously appeared. Our starting point is (iii'). We sub-divide $Q$ dyadically, to select a family of non-overlapping cubes $\left\{Q_{j}\right\}$ which are maximal with respect to the property that

$$
\Re e \frac{1}{\left|Q_{j}\right|} \int_{Q_{j}} b_{Q} \leq 1 / 2 .
$$

If $E_{Q}^{*}$ is defined as in (4.11) with respect to this family $\left\{Q_{j}\right\}$, then by construction, if $(x, t) \in E_{Q}^{*}$, it follows that

$$
\Re e A_{t} b_{Q}(x) \geq 1 / 2
$$

so that (4.12) holds. It remains only to verify that there exists $\eta>0$ such that

$$
\left|E_{Q}\right| \geq \eta|Q|
$$

where $E_{Q}:=Q \backslash\left(\bigcup_{j} Q_{j}\right)$. By (iii') we have that

$$
\begin{aligned}
|Q| & =\int_{Q} b_{Q}=\Re e \int_{Q} b_{Q}=\Re e \int_{E_{Q}} b_{Q}+\Re e \sum_{j} \int_{Q_{j}} b_{Q} \\
& \leq\left|E_{Q}\right|^{\frac{1}{2}}\left(\int_{Q}\left|b_{Q}\right|^{2}\right)^{\frac{1}{2}}+\frac{1}{2} \sum\left|Q_{j}\right|,
\end{aligned}
$$

when in the last step we have used (4.13). From hypothesis (i), we then obtain

$$
|Q| \leq \sqrt{C_{0}}\left|E_{Q}\right|^{\frac{1}{2}}|Q|^{\frac{1}{2}}+\frac{1}{2}|Q|
$$

and (4.14) now follows readily with $\eta=\frac{1}{4 C_{0}}$. 
To conclude the proof, we apply Lemma 4.6 with

$$
\begin{aligned}
& d \mu=\left|\Theta_{t} 1(x)\right|^{2} \frac{d x d t}{t} \quad \text { and } \\
& C_{1}=4 C_{2} \sup _{Q \in \mathbb{D}} \frac{1}{|Q|} \iint_{R_{Q}}\left|\left(\Theta_{t} 1\right) A_{t} b_{Q}\right|^{2} \frac{d x d t}{t} .
\end{aligned}
$$

The previous Theorem has an extension to the matrix valued setting. We explain in the next subsection why this is interesting. Let $\mathbb{M}^{N}$ denote the space of $N \times N$ matrices with complex entries.

Theorem 4.15 Suppose that $\Psi_{t}: \mathbb{R}^{n} \times \mathbb{R}^{n} \rightarrow \mathbb{C}^{N}$ satisfies the standard kernel conditions (1.14), (1.15). Define, for $\mathbf{f}: \mathbb{R}^{n} \rightarrow \mathbb{C}^{N}$, the operator

$$
\boldsymbol{\Theta}_{\mathbf{t}} \cdot \mathbf{f}(x):=\int \boldsymbol{\Psi}_{t}(x, y) \cdot \mathbf{f}(y) d y .
$$

Suppose also that there are constants $\delta>0, C_{0}<\infty$ and a system of matrix valued functions $\mathbf{b}_{Q}: \mathbb{R}^{n} \rightarrow \mathbb{M}^{N}$, indexed by the dyadic cubes, such that

(i) $\int_{\mathbb{R}^{n}}\left|\mathbf{b}_{Q}(x)\right|^{2} d x \leq C_{0}|Q|$;

(ii) $\int_{0}^{\ell(Q)} \int_{Q}\left|\boldsymbol{\Theta}_{t} \mathbf{b}_{Q}(x)\right|^{2} \frac{d x d t}{t} \leq C_{0}|Q|$;

(iii) Re $\xi \cdot\left(|Q|^{-1} \int_{Q} \mathbf{b}_{Q}(x) d x\right) \xi \geq \delta|\xi|^{2}$.

where the ellipticity condition (iii) holds for all $\xi \in \mathbb{C}^{N}$, and where the action of $\boldsymbol{\Theta}_{t}$ on the matrix valued function $\mathbf{b}_{Q}$ is defined in the obvious way as in (4.16) by viewing the kernel $\Psi_{t}(x, y)$ as a $1 \times N$ matrix which multiplies the $N \times N$ matrix $\mathbf{b}_{Q}$. By $\left|\mathbf{b}_{Q}(x)\right|$ is meant the operator norm of the matrix $\mathbf{b}_{Q}(x)$. Then the following square function estimate holds:

$$
\iint_{\mathbb{R}_{+}^{n+1}}\left|\boldsymbol{\Theta}_{t} \cdot \mathbf{f}\right|^{2} \frac{d x d t}{t} \lesssim\|\mathbf{f}\|_{2}^{2}
$$

Remark 4.18 It turns out that a variant of this theorem lies at the heart of the solution of the Kato problem $[9,56,60]$. See also [18], where a similar result is given but with (3.19) in lieu of (iii). Thus, the present result, along with the scalar version Theorem 4.7, addresses the question posed immediately following Remark 3.18.

We now sketch the proof, which is essentially the same as the argument used to establish the Kato conjecture. Let $\mathbf{1}$ denote the $N \times N$ identity matrix. Since

$$
\boldsymbol{\Theta}_{t} \mathbf{1}=\left(\Theta_{t}^{1} 1, \Theta_{t}^{2} 1, \ldots, \Theta_{t}^{N} 1\right)
$$


Proposition 3.4 therefore implies that it is enough to show that $\left|\boldsymbol{\Theta}_{t} \mathbf{1}\right|^{2} t^{-1} d x d t$ is a Carleson measure. For $\epsilon$ small, but fixed, cover $\mathbb{C}^{N}$ by cones

$$
\Gamma_{k}^{\epsilon}=\left\{z \in \mathbb{C}^{N}:\left|\frac{z}{|z|}-v_{k}\right|<\epsilon\right\}
$$

where $v_{k} \in \mathbb{M}^{N}$ with $\left|v_{k}\right|=1$ for $k=1,2,3, \ldots, K(\epsilon, N)$. We see that

$$
\int_{0}^{\ell(Q)} \int_{Q}\left|\boldsymbol{\Theta}_{t} \mathbf{1}\right|^{2} \frac{d x d t}{t} \leq \sum_{k=1}^{K} \int_{0}^{\ell(Q)} \int_{Q}\left|\boldsymbol{\Theta}_{t} \mathbf{1}\right|^{2} 1_{\Gamma_{k}^{\epsilon}}\left(\boldsymbol{\Theta}_{t} \mathbf{1}\right) \frac{d x d t}{t}
$$

Thus, it suffices to show that there is a constant $C_{1}=C_{1}\left(\epsilon, \delta, C_{0}, n, N\right)$ such that

$$
\sup _{Q \in \mathbb{D}}|Q|^{-1} \int_{0}^{\ell(Q)} \int_{Q}\left|\boldsymbol{\Theta}_{t} \mathbf{1}\right|^{2} 1_{\Gamma^{\epsilon}}\left(\boldsymbol{\Theta}_{t} \mathbf{1}\right) \frac{d x d t}{t} \leq C_{1},
$$

for each fixed cone $\Gamma^{\epsilon}$, provided that $\epsilon$ is small enough, but fixed.

To this end, normalizing so that $\delta=1$, and fixing $Q$, we follow the stopping time argument of the previous theorem, in the present case extracting dyadic subcubes $Q_{j} \subset Q$ which are maximal with respect to the property that at least one of the following holds:

$$
\int_{Q_{j}}\left|\mathbf{b}_{Q}\right| \geq \frac{1}{4 \epsilon}
$$

or

$$
\Re e v \cdot\left(\left|Q_{j}\right|^{-1} \int_{Q_{j}} \mathbf{b}_{Q}\right) \bar{v} \leq \frac{3}{4}
$$

where $v \in \mathbb{C}^{N}$ is the unit vector in the direction of the central axis of $\Gamma^{\epsilon}$, i.e.,

$$
\Gamma^{\epsilon}=\left\{z \in \mathbb{C}^{N}:\left|\frac{z}{|z|}-v\right|<\epsilon\right\}
$$

As in the proof of the previous theorem, one may check that

$$
\left|E_{Q}\right|:=\left|Q \backslash\left(\cup_{j} Q_{j}\right)\right| \geq \eta|Q|,
$$

for some fixed $\eta>0$. Moreover, for $(x, t) \in E_{Q}^{*}:=R_{Q} \backslash\left(\bigcup_{j} R_{Q_{j}}\right)$, and for $z \in \Gamma^{\epsilon}$, we claim that

$$
\left|z \cdot A_{t} \mathbf{b}_{Q}(x) \bar{\nu}\right| \geq \frac{1}{2}|z|,
$$


where again $A_{t}$ denotes the dyadic averaging operator defined in (4.2). Indeed, since the opposite inequalities to (4.19) and (4.20) hold in $E_{Q}^{*}$, we have that

$$
\left|\omega \cdot A_{t} \mathbf{b}_{Q}(x) \bar{\nu}\right| \geq\left|v \cdot A_{t} \mathbf{b}_{Q}(x) \bar{\nu}\right|-\left|(\omega-v) \cdot A_{t} \mathbf{b}_{Q}(x) \bar{\nu}\right| \geq \frac{3}{4}-\frac{1}{4}=\frac{1}{2}
$$

when $|\omega-\nu|<\epsilon$ and $(x, t) \in E_{Q}^{*}$. Taking $\omega=z /|z|$ with $z \in \Gamma^{\epsilon}$, we obtain (4.21).

Consequently, we have that

$$
\iint_{E_{Q}^{*}}\left|\boldsymbol{\Theta}_{t} \mathbf{1}\right|^{2} 1_{\Gamma^{\epsilon}}\left(\boldsymbol{\Theta}_{t} \mathbf{1}\right) \frac{d x d t}{t} \leq 4 \iint_{E_{Q}^{*}}\left|\boldsymbol{\Theta}_{t} \mathbf{1} \cdot A_{t} \mathbf{b}_{Q} \bar{\nu}\right|^{2} \frac{d x d t}{t},
$$

and the rest of the proof follows as in the previous theorem.

\subsection{Application to the Kato square root problem}

As mentioned above, a variant of the preceding theorem leads to the solution of the Kato problem. We recall the statement of the problem. Let $A$ be an $n \times n$ matrix of complexvalued $L^{\infty}$ coefficients, defined on $\mathbb{R}^{n}$, and satisfying the ellipticity (or accretivity) condition (1.26). Then the associated divergence form operator $L=-\operatorname{div} A \nabla$ defined as in (1.27), considered as an unbounded operator in the Hilbert space $L^{2}\left(\mathbb{R}^{n}\right)$ with inner product $(u, v):=\langle u, \bar{v}\rangle$, has both its spectrum $\sigma(L)$ and its numerical range $\left\{(L u, u) \in \mathbb{C} ; u, \nabla u, L u \in L^{2}\right\}$ contained in a sector $\{\zeta \in \mathbb{C} ;|\arg \zeta| \leq \omega\}$ for some $\omega \in[0, \pi / 2)$. Such an operator, called $\omega$-accretive, generates a contraction semigroup $\left\{e^{-t L}\right\}_{t>0}$ and has unique $\alpha \omega$-accretive fractional powers $L^{\alpha}$ when $0<\alpha<1$. In particular, $L$ has a unique square root $\sqrt{L}:=L^{1 / 2}$ satisfying $\sqrt{L} \sqrt{L}=L$. See $[69,68]$.

We note for later use (see e.g. [8]) that such an $\omega$-accretive operator $L$ also satisfies the uniform bounds

$$
\left\|\tau L e^{-\tau L} u\right\|_{2} \lesssim\|u\| \quad \text { for all } \tau>0
$$

and (since $L$ is one-one) quadratic estimates such as

$$
\int_{0}^{\infty}\left\|\sqrt{\tau L} e^{-\tau L} u\right\|^{2} \frac{d \tau}{\tau} \approx\|u\|^{2}
$$

When $A$, and hence $L$, is self-adjoint, then it is easy to see that the domain of the operator $\sqrt{L}$ is the Sobolev space $W^{1,2}\left(\mathbb{R}^{n}\right)$, because

$$
\|\sqrt{L} u\|_{2}=(L u, u)^{1 / 2}=(A \nabla, \nabla u)^{1 / 2} \approx\|\nabla u\|_{2} .
$$

The Kato square root problem is to establish the same equivalence of norms

$$
\|\sqrt{L} u\|_{2} \approx\|\nabla u\|_{2}
$$


for non self-adjoint operators, with $C$ depending only on $n, \lambda$ and $\Lambda$. When the dimension $n=1$, the latter estimate is closely related to the $L^{2}$ boundedness of the Cauchy singular integral operator on a Lipschitz curve, and indeed it was solved affirmatively in the same paper [39].

The initial affirmative results for $n \geq 2$ concerned small perturbations of the Laplacian, i.e. $\|A-I\|_{\infty}<\epsilon$ for some small $\epsilon[30,49,66]$. After that came various partial results (see [74] for a survey up to this point), but the main achievement for a long time after was the writing of the book [18] by Auscher and Tchamitchian, consolidating and extending prior results, and relating them to local $T b$ square function estimates (cf. Remark 4.18.) This led on to affirmative solutions, first in 2 dimensions [60], then in all dimensions for small perturbations of real symmetric operators [10], and for operators which satisfy Gaussian heat kernel bounds [56], and finally for all divergence form operators with bounded measurable coefficients [9].

In order to give some feel for the connection with local $T b$ theorems for square functions, let us make the additional assumption of heat kernel bounds $(\mathrm{G})$ :

$$
e^{-\tau L} u(x)=\int_{\mathbb{R}^{n}} k_{\tau}(x, y) u(y) d y \quad \text { for all } u \in L^{2}\left(\mathbb{R}^{n}\right)
$$

where the heat kernel $k_{\tau}(x, y)$ satisfies the Gaussian kernel bounds:

$$
\begin{aligned}
& \left|k_{\tau}(x, y)\right| \leq \frac{\beta}{t^{n / 2}} e^{-\frac{|x-y|^{2}}{\alpha \tau}} \text { and } \\
& \left|k_{\tau}(x+h, y)-k_{\tau}(x, y)\right|+\left|k_{\tau}(x, y+h)-k_{\tau}(x, y)\right| \leq \beta \frac{|h|^{\alpha}}{t^{(\alpha+n) / 2}} e^{-\frac{|x-y|^{2}}{\alpha \tau}} \quad \forall|h| \leq t,
\end{aligned}
$$

for some $\alpha, \beta>0$. We remark that the classes of operators which are stated to satisfy Poisson kernel bounds in the paragraph following equation (1.28), also satisfy heat kernel bounds.

What follows is an outline of the proof under this additional assumption, essentially following the relevant parts of the book [18] and the paper [56].

As $L^{*}$ has the same form as $L$, only the direction

$$
\|\sqrt{L} u\|_{2} \lesssim\|\nabla u\|_{2}
$$

needs be shown, because $\left\|\sqrt{L^{*}} u\right\|_{2} \leq C\|\nabla u\|_{2}$ implies that $\|\nabla u\|_{2} \lesssim\|\sqrt{L} u\|_{2}$, for

$$
\|\nabla u\|_{2}^{2} \leq \frac{1}{\lambda}(A \nabla u, \nabla u)=\frac{1}{\lambda}\left(\sqrt{L} u, \sqrt{L^{*}} u\right) \leq \frac{C}{\lambda}\|\sqrt{L} u\|_{2}\|\nabla u\|_{2} .
$$

(We are leaving out technical considerations concerning domains of operators, etc.)

Now (4.25) is equivalent to the square function estimate:

$$
\iint_{\mathbb{R}_{+}^{n+1}}\left|t L e^{-t^{2} L} u(x)\right|^{2} \frac{d x d t}{t} \lesssim\|\nabla u\|_{2}^{2},
$$


because, by (4.23),

$$
\iint_{\mathbb{R}_{+}^{n+1}}\left|t L e^{-t^{2} L} u(x)\right|^{2} \frac{d x d t}{t}=\frac{1}{2} \int_{0}^{\infty}\left\|\sqrt{\tau L} e^{-\tau L}(\sqrt{L} u)\right\|_{2}^{2} \frac{d \tau}{\tau} \approx\|\sqrt{L} u\|_{2}^{2} .
$$

The square function estimate (4.26) has the form of equation (4.17) in Theorem 4.15 with

$$
\boldsymbol{\Theta}_{t}=t e^{-t^{2} L} \operatorname{div} A
$$

and $\mathbf{f}=\nabla u$. However we cannot apply this theorem as stated because $\boldsymbol{\Theta}_{t}$ does not satisfy the standard kernel estimates (1.14) and (1.15), and indeed the square function estimate (4.17) can, in this case, only be expected to hold for gradient vector fields $\mathbf{f}=\nabla u$. To proceed, the structure of the operator $L$ is used to show that the operators $\left\{Z_{t}\right\}$, defined by

$$
\begin{aligned}
Z_{t} u(x): & =\boldsymbol{\Theta}_{t} \nabla u(x)-\Theta_{t} \mathbf{1}(x) \cdot\left(P_{t} \nabla u\right)(x) \\
& =\left(t L e^{-t^{2} L} u\right)(x)-\left(t L e^{-t^{2} L} \boldsymbol{\phi}(x)\right) \cdot\left(P_{t} \nabla u\right)(x),
\end{aligned}
$$

(where $\phi(x):=x$ ) satisfy

$$
\iint_{\mathbb{R}_{+}^{n+1}}\left|Z_{t} u(x)\right|^{2} \frac{d x d t}{t} \lesssim\|\nabla u\|_{2}^{2}
$$

Once we have proved that $d \mu(x):=\left|\boldsymbol{\Theta}_{t} \mathbf{1}(x)\right|^{2} \frac{d x d t}{t}$ is a Carleson measure on $\mathbb{R}_{+}^{n+1}$, we can then verify (4.26) as follows:

$$
\begin{gathered}
\iint_{\mathbb{R}_{+}^{n+1}}\left|t L e^{-t^{2} L} u(x)\right|^{2} \frac{d x d t}{t}=\iint_{\mathbb{R}_{+}^{n+1}}\left|Z_{t} u(x)+\boldsymbol{\Theta}_{t} \mathbf{1}(x) \cdot\left(P_{t} \nabla u\right)(x)\right|^{2} \frac{d x d t}{t} \\
\quad \lesssim \iint_{\mathbb{R}_{+}^{n+1}}\left|Z_{t} u(x)\right|^{2} \frac{d x d t}{t}+\iint_{\mathbb{R}_{+}^{n+1}}\left|P_{t} \nabla u(x)\right|^{2}\left|\Theta_{t} \mathbf{1}(x)\right|^{2} \frac{d x d t}{t} \lesssim\|\nabla u\|_{2},
\end{gathered}
$$

by (4.27) and Carleson's Theorem as used in (3.9). (We shall not include a proof of (4.27), though remark that it is somewhat similar to the " $T 1$ "-type reductions of square function bounds to Carleson measure bounds which we have already considered.)

The next step is to proceed exactly as in Theorem 4.15, to show that the Carleson estimate is a consequence of an estimate of the form:

$$
\iint_{R_{Q}}\left|\Theta_{t} \mathbf{1}(x) \cdot A_{t} \mathbf{b}_{Q}(x)\right|^{2} \frac{d x d t}{t} \leq C_{2}|Q| \text { for all dyadic cubes } Q \subset \mathbb{R}^{n},
$$

provided we can find a system of matrix valued functions $\mathbf{b}_{Q}: \mathbb{R}^{n} \rightarrow \mathbb{M}^{N}, Q \in \mathbb{D}$, which satisfies hypotheses (i) and (iii) of that theorem. For this purpose, we define $\mathbf{b}_{Q}: \mathbb{R}^{n} \rightarrow \mathbb{M}^{N}$ for each dyadic cube $Q \subset \mathbb{R}^{n}$ by 


$$
\mathbf{b}_{Q}=\nabla e^{-\epsilon^{2} \ell(Q)^{2} L} \boldsymbol{\phi}_{Q}=: \nabla \mathbf{f}_{Q},
$$

with $\epsilon>0$ to be chosen, small enough, and

$$
\boldsymbol{\phi}_{Q}(x)=\eta_{Q}(x)\left(x-x_{Q}\right),
$$

where $\eta_{Q} \in C_{0}^{\infty}(5 Q)$ with $\eta \equiv 1$ on $Q$, and $x_{Q}$ denotes the centre of $Q$.

Before checking the hypotheses (i)-(iii), we note some consequences of the heat kernel bounds $(\mathrm{G})$, namely that $\boldsymbol{\Theta}_{t} \mathbf{1} \in L^{\infty}\left(\mathbb{R}^{n}, \mathbb{C}^{n}\right)$ with a uniform bound

$$
\begin{array}{cc}
\left\|\boldsymbol{\Theta}_{t} \mathbf{1}\right\|_{\infty}=\left\|t L e^{-t^{2} L} \boldsymbol{\phi}\right\|_{\infty} \leq M_{1}<\infty & \text { for all } t>0 ; \text { and } \\
\left\|\left(e^{-t^{2} L}-I\right) \boldsymbol{\phi}_{Q}\right\|_{\infty} \leq M_{2} t & \text { for all } t>0 .
\end{array}
$$

We now show that the hypotheses (i), (ii), (iii) of Theorem 4.15 are satisfied by the system of matrix valued functions $\mathbf{b}_{Q}$.

(i) The estimate $\left\|\mathbf{b}_{Q}\right\|_{2}^{2} \leq C_{0}|Q|$ is a consequence of the more general fact that a homogeneous elliptic operator $L$ in divergence form generates a bounded semigroup with respect to the homogeneous Sobolev norm $\|\nabla u\|_{2}$. Thus

$$
\left\|\mathbf{b}_{Q}\right\|_{2}=\left\|\nabla e^{-\epsilon^{2} \ell(Q)^{2} L} \boldsymbol{\phi}_{Q}\right\|_{2} \lesssim\left\|\nabla \boldsymbol{\phi}_{Q}\right\|_{2} \approx|Q|^{1 / 2} .
$$

(ii) Noting that $\boldsymbol{\Theta}_{t} \mathbf{b}_{Q}(x)=t e^{-t^{2} L} L e^{-\epsilon^{2} \ell(Q)^{2} L} \boldsymbol{\phi}_{Q}$, we have

$$
\begin{aligned}
\int_{0}^{\ell(Q)} \int_{Q}\left|\boldsymbol{\Theta}_{t} \mathbf{b}_{Q}(x)\right|^{2} \frac{d x d t}{t} \leq & \int_{0}^{\infty}\left\|L e^{-\left(t^{2}+\epsilon^{2} \ell(Q)^{2}\right) L} \boldsymbol{\phi}_{Q}\right\|_{2}^{2} t d t \\
= & \frac{1}{2} \int_{\epsilon^{2} \ell(Q)^{2}}^{\infty}\left\|\tau L e^{-\tau L} \boldsymbol{\phi}_{Q}\right\|_{2}^{2} \frac{d \tau}{\tau^{2}} \\
& \text { where } \tau=t^{2}+\epsilon^{2} \ell(Q)^{2} \\
\leq & c_{0}\left(\frac{1}{\epsilon^{2} \ell(Q)^{2}}\right)\left\|\boldsymbol{\phi}_{Q}\right\|_{2}^{2} \leq \frac{c_{1}}{\epsilon^{2}}|Q|,
\end{aligned}
$$

using (4.22) to obtain the uniform operator bounds.

(iii)

$$
\begin{aligned}
\Re e \xi \cdot\left(|Q|^{-1} \int_{Q} \mathbf{b}_{Q}(x) d x\right) \bar{\xi} & =|\xi|^{2}+\Re e \xi \cdot\left(|Q|^{-1} \int_{Q}\left(\mathbf{b}_{Q}-\mathbf{1}\right)(x) d x\right) \bar{\xi} \\
& \geq|\xi|^{2}-|Q|^{-1}\left\|\int_{Q} \nabla\left(e^{-\epsilon^{2} \ell(Q)^{2} L}-I\right) \boldsymbol{\phi}_{Q}(x) d x\right\|_{o p}|\xi|^{2} \\
& \geq|\xi|^{2}-\frac{c_{1}}{\ell(Q)}\left\|\left(e^{-\epsilon^{2} \ell(Q)^{2} L}-I\right) \boldsymbol{\phi}_{Q}\right\|_{\infty}|\xi|^{2} \\
& \geq|\xi|^{2}\left(1-c_{1} M_{2} \epsilon\right) \quad \text { (by (4.31)) } \\
& \geq \frac{1}{2}|\xi|^{2}
\end{aligned}
$$


provided $\epsilon$ is chosen sufficiently small. We fix such a value of $\epsilon$, and in particular use it back in part (ii).

All that remains is to check (4.28). To this end, as in the proofs of Theorems 4.7 and 4.15 above, we follow [38] to write

$$
\boldsymbol{\Theta}_{t} \mathbf{1} \cdot\left(A_{t} \mathbf{b}_{Q}\right)=\boldsymbol{\Theta}_{t} \mathbf{1} \cdot\left(\left(A_{t}-P_{t}\right) \mathbf{b}_{Q}\right)+\left(\boldsymbol{\Theta}_{t} \mathbf{1} \cdot P_{t}-\boldsymbol{\Theta}_{t}\right) \mathbf{b}_{Q}+\boldsymbol{\Theta}_{t} \mathbf{b}_{Q},
$$

and we note that, in view of (4.29), $\left(\boldsymbol{\Theta}_{t} \mathbf{1} \cdot P_{t}-\boldsymbol{\Theta}_{t}\right) \mathbf{b}_{Q}=-Z_{t} \mathbf{f}_{Q}$. Thus,

$$
\iint_{R_{Q}}\left|\boldsymbol{\Theta}_{t} \mathbf{1}(x) \cdot\left(A_{t} \mathbf{b}_{Q}\right)(x)\right|^{2} \frac{d x d t}{t} \lesssim\left\|\mathbf{b}_{Q}\right\|_{2}^{2}+\left\|\mathbf{b}_{Q}\right\|_{2}^{2}+C_{0}|Q| \leq C_{2}|Q|
$$

by (4.30), (4.4), (4.27) and (ii).

That completes our description of the proof of the Kato square root problem for elliptic operators which satisfy pointwise heat kernel bounds. As we have said, the result holds without this additional assumption. One may use, in lieu of the Gaussian heat kernel bounds $(\mathrm{G})$, the "Davies-Gaffney" off-diagonal estimates, which hold for every divergence form elliptic operator $L$ as in (1.25)-(1.27). We refer the reader to [9] for a complete proof of the Kato square root estimate in this general setting.

The question whether accretive operators satisfy the estimate (4.24) was originally asked by T. Kato, J.-L. Lions and others in an attempt to better understand the equivalence between the operators and their associated sesquilinear (or energy) forms. This question arose again during Kato's study of hyperbolic wave equations with time-varying coefficients, as it is connected with the question whether the mapping $A \rightarrow L^{1 / 2}$ is analytic. See [72]. Another application, and an easier one to describe, was noted by Kenig [70, Remark 2.5.6]: On $\mathbb{R}_{+}^{n+1}:=\mathbb{R}^{n} \times(0, \infty)$ consider the Dirichlet problem:

$$
\left\{\begin{aligned}
\frac{\partial^{2}}{\partial t^{2}} U(\cdot, t)-L U(\cdot, t) & =0 \\
U(\cdot, t) & =u(\cdot) \in \mathcal{D}(\sqrt{L})
\end{aligned}\right.
$$

where still $L=-\sum_{j, k=1}^{n} \frac{\partial}{\partial x_{j}}\left(A_{j k} \frac{\partial}{\partial x_{k}}\right)$. Then the solution $U(x, t):=e^{-t \sqrt{L}} u(x)$ also satisfies the Neumann boundary condition

$$
\left.\frac{\partial U}{\partial t}\right|_{t=0}=-\sqrt{L} u
$$

if $\sqrt{L} u \in L^{2}\left(\mathbb{R}^{n}\right)$. Hence the Kato estimate $\|\sqrt{L} u\|_{2} \approx\left\|\nabla_{x} u\right\|_{2}$ is equivalent to

$$
\left\|\left.\frac{\partial U}{\partial t}\right|_{t=0}\right\|_{2} \approx\left\|\nabla_{x} u\right\|_{2}
$$

This alternative form of the Kato estimate is also known as a Rellich inequality or a Dirichlet-Neumann inequality.

For an excellent survey of the Kato square root problem, see Kenig's featured review [71]. 
Before completing this section, we comment briefly on $L^{p}$ estimates. For the various $T b$ results proved up to Section 4.1, the estimates also hold with $L^{p}$ norms in place of the $L^{2}$ norms, as follows from Theorem 2.1 (to treat the case $2<p<\infty$, this requires either that we work with conical square functions, or that we impose some regularity in the $x$-variable, of the kernel $\psi_{t}(x, y)$; see, e.g., [11] for a more detailed discussion of this point.) On the other hand, the operators $\boldsymbol{\Theta}_{t}$ used in the current section, arising in the proof of Kato's square root estimate, do not typically satisfy standard kernel bounds, even if $L$ does have heat kernel bounds, so we cannot expect the Kato estimates to remain true for all $p \neq 2$. However, a variety of means have been used to prove bounds in specific cases. One method, involving weak-type (1-1) bounds via an adaptation of the Calderón-Zygmund theorem [46], was used to obtain

$$
\|\nabla u\|_{p} \lesssim\|\sqrt{L} u\|_{p}
$$

when $1<p<2$, provided $L$ has pointwise heat kernel bounds (i.e. satisfies the first equation in (G)) [47], and, via a duality argument,

$$
\|\sqrt{L} u\|_{p} \lesssim\|\nabla u\|_{p}
$$

when $2<p<\infty$.Another, involving the use of Hardy spaces, was used in [9] to show that if $L$ has pointwise heat kernel bounds, then (4.33) holds when $1<p<2$. In the absence of pointwise kernel bounds, an extension of the Calderón-Zygmund method of [46] was developed independently in [20,21], and in [57], to prove the "Riesz transform" estimate (4.32) for a (necessarily) restricted range of $p$; the Kato estimate (4.33) was proved for a sharp (and again restricted) range of $p$ in [3]. We refer the reader to [3] for a rather complete discussion of the $L^{p}$ theory in the absence of pointwise kernel bounds. Another approach to $L^{p}$ estimates is mentioned in Section 5.1.3 below.

\section{Further results and recent progress}

In this section we briefly discuss some recent advances in this subject.

\section{$5.1 \mathrm{~Tb}$ theory for SIOs}

\subsubsection{Analytic capacity}

As mentioned above, Christ's local $T b$ theorem (Theorem 4.1) was motivated in part by its connection with the theory of analytic capacity and the Painlevé problem, which was eventually solved in the remarkable work of Tolsa [88]. See also the earlier work of Mattila, Melnikov and Verdera [77], and David [41,42]. Analytic capacity is connected with $T b$ theory via the Cauchy integral: the existence of non-constant bounded analytic functions may be used to produce a testing function $b$ which yields $L^{2}$ bounds for the Cauchy integral. These bounds, in turn, encode geometric information via the so-called Menger curvature. In practice, this program was quite difficult to carry out, especially 
in the general situation in which the underlying measure may be non-doubling. Some extensions of either local or global $T b$ theorems to the non-doubling setting have been obtained by David [41] and by Nazarov, Treil and Volberg [79,80]. The latter, especially, played a useful role in Tolsa's solution of the Painlevé problem and the related Vitushkin conjecture concerning the semi-additivity of analytic capacity. Finally, much of this theory has been extended to higher dimensions by Volberg [90].

\subsubsection{Local Tb theory for SIOs}

Theorem 4.1 has been extended in another direction, closer in spirit to the results that we described above in Section 4 , in which one requires weaker quantitative control on $b_{Q}$ and $T b_{Q}$. In [13], Conditions (i) and (iii) of Theorem 4.1 are relaxed to

$$
\text { (i) } \int_{Q}\left|b_{Q}^{i}\right|^{p} \leq C_{0}|Q|, \quad \text { (ii) } \int_{Q}\left|T b_{Q}^{1}\right|^{p^{\prime}} \leq C_{0}|Q|,\left.\left.\int_{Q}\right|^{t} T b_{Q}^{2}\right|^{p^{\prime}} \leq C_{0}|Q|,
$$

respectively, for $1<p<\infty$, assuming that $T$ is a perfect dyadic SIO. In the case of standard SIOs, the same result was obtained with $p=2$ in [19], but it remains an open problem, in general, to treat the case $1<p<2$. The current state of the art appears in [17], where the case $1<p<2$ is handled in the presence of additional hypotheses in the spirit of WBP, and in [62], where the theory is extended to a class of non-doubling measures.

\subsubsection{Tb theory for vector valued functions}

Many applications require the study of SIOs acting on spaces of vector valued functions $f: \mathbb{R}^{n} \rightarrow X$, where $X$ is a Banach space. As mentioned in the Introduction, many proofs in this survey carry over to this context provided $X$ is a Hilbert space. However, the situation is much more complicated when $X$ is not isomorphic to a Hilbert space, and even the Hilbert transform may then be unbounded. In the 1980's, Bourgain [22] and Burkholder [23] proved that the Hilbert transform is bounded on $L^{2}\left(\mathbb{R}^{n} ; X\right)$ if and only if $X$ has the UMD (Unconditional Martingale Difference) property. See [24] for a survey. The connection between SIOs and martingales turns out to be the key to the vector valued theory, but is also an important tool for the scalar valued theory, for example in obtaining best constants. The first UMD valued $T 1$ theorem was obtained in the influential paper [48] by Figiel. Over the past ten years, applications to PDEs such as maximal regularity, have motivated the study of SIOs with operator valued kernels $K: \mathbb{R}^{n} \times \mathbb{R}^{n} \backslash\{x=y\} \rightarrow B(X)$, where $B(X)$ denotes the set of bounded linear operators from $X$ to itself, and $X$ is a UMD space. In this direction, a $T 1$ theorem was obtained by Hytönen and Weis [64], and a $T b$ theorem followed in [65]. A type of local $T b$ theorem for square functions, used to solve Kato's problem in $L^{p}\left(\mathbb{R}^{n}\right)$ and more generally in a UMD valued context, was then proven in [63]. Currently the vector valued theory is also being developed in contexts where the space of variables $\mathbb{R}^{n}$ is replaced by a more general metric measure space; see [61]. 


\subsection{Local $T b$ theory for square functions and applications}

\subsection{1 $L^{p}$ control on $b_{Q}$}

In contrast to the situation for SIOs, the local $T b$ theory for square functions has been generalized to permit $b_{Q} \in L^{p}$, with $1<p<2$, as in (5.1.2). See [55], where this is done in the Euclidean case (i.e., to obtain the square function estimate (4.8) in the upper half space $\mathbb{R}_{+}^{n+1}$ ). A further extension to the case that the half-space is replaced by $\mathbb{R}^{n+1} \backslash E$, where $E$ is a closed Ahlfors-David regular set of Hausdorff dimension $n$, appears in [52]. The latter extension has been used to prove a result of free boundary type, in which higher integrability of the Poisson kernel, in the presence of certain natural background hypotheses, is shown to be equivalent to a quantitative rectifiability of the boundary $[58,59]$. In the spirit of the work mentioned above on analytic capacity, local $\mathrm{Tb}$ theory enters by allowing one to relate Poisson kernel estimates to square function bounds for harmonic layer potentials, which in turn are tied to quantitative rectifiability of the boundary. In this case, the " $b_{Q}$ 's" are normalized Poisson kernels.

\subsubsection{Extensions of the Kato problem and elliptic PDEs}

The circle of ideas involved in the solution of the Kato problem, including local $T b$ theory for square functions, has been used to establish certain generalizations of the Kato problem with applications to complex elliptic PDEs and systems. These include $L^{2}$ bounds for layer potentials associated to complex divergence form elliptic operators [4,55], and the development of an $L^{2}$ functional calculus of certain perturbed Dirac operators and other first order elliptic systems [5-7,14]. The layer potential bounds, and the existence of a bounded holomorphic functional calculus for first order elliptic systems, were each then applied to obtain $L^{2}$ solvability results for elliptic boundary value problems.

The local $T b$ theory for square functions has also been used to establish other generalizations of the Kato problem, such as to higher order elliptic operators and systems [12] and to elliptic operators on Lipschitz domains [15].

Acknowledgments Some of the material on local $T b$ theory for square functions is taken from the first named author's ICM lecture [54]. The second author thanks Pierre Portal for helpful contributions to the manuscript, and to Maren Schmalmack for the diagram. Both authors wish to express their appreciation to Pascal Auscher with whom we have maintained a long and fruitful collaboration on topics covered by this survey.

Open Access This article is distributed under the terms of the Creative Commons Attribution License which permits any use, distribution and reproduction in any medium, provided the original author(s) and source are credited.

\section{References}

1. Auscher, P.: Regularity theorems and heat kernel for elliptic operators. J. Lond. Math. Soc. (2) 54(2), 284-296 (1996) 
2. Auscher, P.: Lectures on the Kato square root problem. Surveys in analysis and operator theory (Canberra, 2001), pp. 1-18. Proc. Centre Math. Appl. Austral. Nat. Univ., vol. 40. Austral. Nat. Univ., Canberra (2002)

3. Auscher, P.: On necessary and sufficient conditions for $L^{p}$-estimates of Riesz transforms associated with elliptic operators on $\mathbb{R}^{n}$ and related estimates. Mem. Am. Math. Soc. 186, 871, xviii+75 (2007)

4. Alfonseca, M., Auscher, P., Axelsson, A., Hofmann, S., Kim, S.: Analyticity of layer potentials and $L^{2}$ solvability of boundary value problems for divergence form elliptic equations with complex $L^{\infty}$ coefficients. Adv. Math. 226(5), 4533-4606 (2011)

5. Auscher, P., Axelsson, A., Hofmann, S.: Functional calculus of Dirac operators and complex perturbations of Neumann and Dirichlet problems. J. Funct. Anal. 255, 374-448 (2008)

6. Auscher, P., Axelsson, A., McIntosh, A.: Solvability of elliptic systems with square integrable boundary data. Ark. Mat. 48, 253-287 (2010)

7. Auscher, P., Axelsson, A., McIntosh, A.: On a quadratic estimate related to the Kato conjecture and boundary value problems. Contemp. Math. AMS 505, 105-129 (2010)

8. Albrecht, D., Duong, X., McIntosh, A.: Operator theory and harmonic analysis. Workshop on Analysis and Geomerty 1995, Part III. Proc. of the CMA, ANU, Canberra, vol. 34, pp. 77-136 (1996)

9. Auscher, P., Hofmann, S., Lacey, M., McIntosh, A., Tchamitchian, Ph.: The solution of the Kato square root problem for second order elliptic operators on $\mathbb{R}^{n}$. Ann. Math. 156, 633-654 (2002)

10. Auscher, P., Hofmann, S., Lewis, J.L., Tchamitchian, Ph.: Extrapolation of Carleson measures and the analyticity of Kato's square root operators. Acta Math. 187(2), 161-190 (2001)

11. Auscher, P., Hofmann, S., Martell, J.M.: Vertical versus conical square functions. Preprint

12. Auscher, P., Hofmann, S., McIntosh, A., Tchamitchian, Ph.: The Kato square root problem for higher order elliptic operators and systems on $\mathbb{R}^{n}$. J. Evol. Equ. 1(4), 361-385 (2001)

13. Auscher, P., Hofmann, S., Muscalu, C., Tao, T., Thiele, C.: Carleson measures, trees, extrapolation, and $T(b)$ theorems. Publ. Mat. 46(2), 257-325 (2002)

14. Axelsson, A., Keith, S., McIntosh, A.: Quadratic estimates and functional calculi of perturbed dirac operators. Invent. Math. 163, 455-497 (2006)

15. Axelsson, A., Keith, S., McIntosh, A.: The Kato square root problem for mixed boundary value problems. J. Lond. Math. Soc. 74(2), 113-130 (2006)

16. Auscher, P., McIntosh, A., Tchamitchian, Ph.: Heat kernels of second order complex elliptic operators and applications. J. Funct. Anal. 152, 22-73 (1998)

17. Auscher, P., Routin, E.: Local $T b$ theorems and Hardy inequalities. J. Geom. Anal., Online: doi:10. 1007/s12220-011-9249-1

18. Auscher, P., Tchamitchian, Ph.: Square root problem for divergence operators and related topics. Astérisque 249. Société Mathématique de France (1998)

19. Auscher, P., Yang, Q.X.: On local T(b) theorems. Publ. Math. 53, 179-196 (2009)

20. Blunck, S., Kunstmann, P.: Calderón-Zygmund theory for non-integral operators and the $H^{\infty}$ functional calculus. Rev. Mat. Iberoamericana 19(3), 919-942 (2003)

21. Blunck, S., Kunstmann, P.: Weak type $(p, p)$ estimates for Riesz transforms. Math. Z. 247, 137-148 (2004)

22. Bourgain, J.: Some remarks on Banach spaces in which martingale difference sequences are unconditional. Ark. Mat. 21, 163-168 (1983)

23. Burkholder, D.L.: A geometric condition that implies the existence of certain singular integrals of Banach-space-valued functions. Conference on harmonic analysis in honor of Antoni Zygmund, vol. I, II (Chicago, Ill., 1981). Wadsworth Math. Ser., Wadsworth, Belmont, CA, pp. 270-286 (1983)

24. Burkholder, D.L.: Martingales and singular integrals in Banach spaces. Handbook of the Geometry of Banach Spaces, vol. I, pp. 233-269. North-Holland, Amsterdam (2001)

25. Calderón, A.P.: Commutators of singular integral operators. Proc. Natl. Acad. Sci. USA 53, 1092-1099 (1965)

26. Calderón, A.P.: Algebras of singular integral operators. Proc. Sympos Pure Math., vol. 10, pp. 18-55. AMS, Providence (1967)

27. Calderón, A.P.: Cauchy integrals on Lipschitz curves and related operators. Proc. Natl. Acad. Sci. USA 74, 1324-1327 (1977)

28. Calderón, A.P.: Commutators, singular integrals on Lipschitz curves and applications. In: Proc. of the International Congress of Mathematicians (Helsinki, 1978), pp. 85-96. Acad. Sci. Fennica, Helsinki (1980) 
29. Carleson, L.: Interpolation of bounded analytic functions and the corona problem. Ann. Math. 76, 547-559 (1962)

30. Coifman, R., Deng, D., Meyer, Y.: Domaine de la racine carrée de certains opérateurs différentiels accrétifs. Ann. Inst. Fourier (Grenoble) 33(2), 123-134 (1983)

31. Christ, M.: A T(b) theorem with remarks on analytic capacity and the Cauchy integral. Colloquium Mathematicum LX/LXI, pp. 601-628 (1990)

32. Christ, M.: Lectures on singular integral operators. CBMS Regional Conference Series in Mathematics, 77. Published for the Conference Board of the Mathematical Sciences, Washington, DC. Amer. Math. Soc., Providence (1990)

33. Christ, M., Journé, J.-L.: Polynomial growth estimates for multilinear singular integral operators. Acta Math. 159(1-2), 51-80 (1987)

34. Coifman, R., Jones, P., Semmes, S.: Two elementary proofs of the $L^{2}$ boundedness of Cauchy integrals on Lipschitz curves. J. Am. Math. Soc. 2(3), 553-564 (1989)

35. Coifman, R., Meyer, Y.: On commutators of singular integrals and bilinear singular integrals. Trans. Am. Math. Soc. 212, 315-331 (1975)

36. Coifman, R., Meyer, Y.: Commutateurs d'intégrales singulières et opérateurs multilinéaires. Ann. Inst. Fourier (Grenoble) 28(3), 177-202 (1978)

37. Coifman, R., Meyer, Y.: Au delà des opérateurs pseudo differentiels, Astérisque. Soc. Math. 57 (1978)

38. Coifman, R., Meyer, Y.: Non-linear harmonic analysis and PDE. In: Stein, E.M. (ed.) Beijing Lectures in Harmonic Analysis. Annals of Math. Studies, vol. 112. Princeton University Press (1986)

39. Coifman, R., McIntosh, A., Meyer, Y.: L'intégrale de Cauchy définit un opérateur borné sur $L^{2}$ pour les courbes lipschitziennes. Ann. Math. 116, 361-387 (1982)

40. Calderón, A.P., Zygmund, A.: On the existence of certain singular integrals. Acta Math. 88, 85-139 (1952)

41. David, G.: Unrectifiable 1-sets have vanishing analytic capacity. Rev. Mat. Iberoamericana 14(2), 369479 (1998)

42. David, G.: Analytic capacity, Calderón-Zygmund operators, and rectifiability. Publ. Mat. 43(1), 3-25 (1999)

43. De Giorgi, E.: Sulla differenziabilità e l'analiticità delle estremali degli integrali multipli regolari. (Italian). Mem. Accad. Sci. Torino. Cl. Sci. Fis. Mat. Nat. 3(3), 25-43 (1957)

44. David, G., Journé, J.-L.: A boundedness criterion for generalized Calderón-Zygmund operators. Ann. Math. 120, 371-398 (1984)

45. David, G., Journé, J.-L., Semmes, S.: Opérateurs de Calderón-Zygmund, fonctions para-accrétives et interpolation. Rev. Mat. Iberoamericana 1, 1-56 (1985)

46. Duong, X.T., McIntosh, A.: Singular integral operators with non-smooth kernels on irregular domains. Rev. Mat. Iberoamericana 15, 233-265 (1999)

47. Duong, X.T., McIntosh, A.: The $L^{p}$ boundedness of Riesz transforms associated with divergence form operators. In: Workshop on Analysis and Applications, Brisbane, 1997. Proc. of the CMA, ANU, Canberra, vol. 37, pp. 15-25 (1999)

48. Figiel, T.: Singular integral operators: a martingale approach. Geometry of Banach spaces (Strobl, 1989). In: Müller, P.F.X., Schachermayer, W. (eds.) London Math. Soc. Lecture Note Ser., vol. 158, pp. 95-110. Cambridge University Press, Cambridge (1990)

49. Fabes, E., Jerison, D., Kenig, C.: Multilinear Littlewood-Paley estimates with applications to partial differential equations . Proc. Natl. Acad. Sci. USA 79(18), 5746-5750 (1982)

50. Fabes, E., Jodeit, M., Rivière, N.: Potential techniques for boundary value problems on $C^{1}$-domains. Acta Math. 141, 165-186 (1978)

51. Fefferman, C., Stein, E.M.: $H^{p}$ spaces of several variables. Acta Math. 129(3-4), 137-193 (1972)

52. Grau de la Herran, A., Mourgoglou, M.: Local $T b$ theorems for square functions on Ahlfors-David regular sets. In preparation

53. García-Cuerva, J., Rubio de Francia, J.L.: Weighted Norm Inequalities and Related Topics. NorthHolland Mathematics Studies, vol. 116. Amsterdam (1985)

54. Hofmann, S.:, Local $T b$ Theorems and applications in PDE. Proceedings of the ICM Madrid, vol. II, pp. 1375-1392. European Math. Soc. (2006)

55. Hofmann, S.: A local $T b$ theorem for square functions. Proc. Symp. Pure Math., vol. 79, pp. 175-185. Special volume "Perspectives in Partial Differential Equations, Harmonic Analysis and Applications", in honor of the 70th birthday of V. Maz'ya (2008) 
56. Hofmann, S., Lacey, M., McIntosh, A.: The solution of the Kato problem for divergence form elliptic operators with Gaussian heat kernel bounds. Ann. Math. 156, 623-631 (2002)

57. Hofmann, S., Martell, J.M.: $L^{p}$ bounds for Riesz transforms and square roots associated with second order elliptic operators. Publ. Mat. 47(2), 497-515 (2003)

58. Hofmann, S., Martell, J.M.: Uniform rectifiability and harmonic measure. Preprint

59. Hofmann, S., Martell, J.M., Uriarte-Tuero, I.: Uniform rectifiability and harmonic measure II: Poisson kernels in $L^{p}$ imply uniform rectifiability. In preparation

60. Hofmann, S., McIntosh, A.: The solution of the Kato problem in two dimensions. In: Proceedings of the Conference on Harmonic Analysis and PDE held in El Escorial, Spain in July 2000. Publ. Mat. Vol. extra, pp. 143-160 (2002)

61. Hytönen, T., Martikainen, H.: Non-homogeneous $T b$ theorem and random dyadic cubes on metric measure spaces. Preprint

62. Hytönen, T., Martikainen, H.: On general local $T b$ theorems. Preprint

63. Hytönen, T., McIntosh, A., Portal, P.: Kato's square root problem in Banach spaces. J. Funct. Anal. 254, 675-726 (2008)

64. Hytönen, T., Weis, L.: A $T 1$ theorem for integral transformations with operator-valued kernel. J. Reine Angew. Math. 599, 155-200 (2006)

65. Hytönen, T.: An operator-valued $T b$ theorem. J. Funct. Anal. 234, 420-463 (2006)

66. Journé, J.-L.: Remarks on Kato's square root problem. Conference on Mathematical Analysis (El Escorial 1989). Publ. Math. 35(1), 299-321 (1991)

67. John, F., Nirenberg, L.: On functions of bounded mean oscillation. Comm. Pure Appl. Math. 14, 415-426 (1961)

68. Kato, T.: Fractional powers of dissipative operators. J. Math. Soc. Japan 13, 246-274 (1961)

69. Kato, T.: Perturbation Theory for Linear Operators. Springer, New York (1966)

70. Kenig, C.: Harmonic analysis techniques for second order elliptic boundary value problems, vol. 83. In: CBMS Regional Conference Series in Mathematics. AMS, Providence (1994)

71. Kenig, C.: Featured review: the solution of the Kato square root problem for second order elliptic operators on $\mathbb{R}^{n}$. Math. Rev. MR1933726 (2004c:47096c) (2004)

72. McIntosh, A.: Square roots of operators and applications to hyperbolic PDE's. Miniconference on Operator Theory and PDE. Proc. of the CMA, ANU Canberra, vol. 5, pp. 124-136 (1984)

73. McIntosh, A.: When are singular integral operators bounded? Miniconference on Geometry and PDEs 1985. Proc. of the CMA, ANU Canberra, vol. 10, pp. 141-149 (1985)

74. McIntosh, A.: The square root problem for elliptic operators. Functional Analytic Methods for Partial Differential Equation. Lecture Notes in Mathematics, vol. 1450, pp. 122-140. Springer, Berlin (1990)

75. McIntosh, A., Meyer, Y.: Algèbres d'opérateurs définis par des intégrales singulières. C. R. Acad. Sci. Paris 301(Série 1), 395-397 (1985)

76. Mikhlin, S.G.: Multidimensional singular integrals and integral equations. Translated from the Russian by W.J.A. Whyte. Pergamon Press, Oxford (1965)

77. Mattila, P., Melnikov, M., Verdera, J.: The Cauchy integral, analytic capacity, and uniform rectifiability. Ann. Math. (2) 144(1), 127-136 (1996)

78. Nash, J.: Continuity of solutions of parabolic and elliptic equations. Am. J. Math. 80, 931-954 (1958)

79. Nazarov, F., Treil, S., Volberg, A.: Accretive system $T b$-theorems on nonhomogeneous spaces. Duke Math. J. 113(2), 259-312 (2002)

80. Nazarov, F., Treil, S., Volberg, A.: The $T b$-theorem on non-homogeneous spaces. Acta Math. 190(2), 151-239 (2003)

81. Peetre, J.: On convolution operators leaving $L^{p, \lambda}$ spaces invariant. Ann. Mat. Pura Appl. (4) 72, 295304 (1966)

82. Semmes, S.: Square function estimates and the $T(b)$ Theorem. Proc. Am. Math. Soc. 110(3), 721-726 (1990)

83. Spanne, S.: Sur l'interpolation entre les espaces $\mathcal{L}_{k} p \Phi$. Ann. Scuola Norm. Sup. Pisa (3) 20, 625-648 (1966)

84. Stein, E.M.: Singular integrals, harmonic functions, and differentiability properties of functions of several variables. Singular integrals. Proc. Sympos. Pure Math., Chicago, 1966, pp. 316-335. Amer. Math. Soc., Providence (1967)

85. Stein, E.M.: Topics in harmonic analysis related to the Littlewood-Paley theory. Annals of Mathematics Studies, No. 63. Princeton University Press, Princeton; University of Tokyo Press, Tokyo (1970) 
86. Stein, E.M.: Singular Integrals and Differentiability Properties of Functions. Princteon University Press, Princeton (1970)

87. Stein, E.M.: Harmonic analysis: real-variable methods, orthogonality, and oscillatory integrals, Princeton Mathematical Series, 43. Monographs in Harmonic Analysis, III. Princeton University Press, Princeton (1993)

88. Tolsa, X.: Painlevé's problem and the semiadditivity of analytic capacity. Acta Math. 190(1), 105-149 (2003)

89. Verchota, G.: Layer potentials and regularity for the Dirichlet problem for Laplace's equation in Lipschitz domains. J. Funct. Anal. 59(3), 572-611 (1984)

90. Volberg, A.: Calderón-Zygmund capacities and operators on nonhomogeneous spaces. In: CBMS Regional Conference Series in Mathematics, vol. 100. Published for the Conference Board of the Math. Sciences, Washington, DC. Amer. Math. Soc., Providence (2003) 